\title{
DIFFERENTIAL OPERATORS PRESERVING RELATIONS OF AUTOMORPHY
}

\author{
BY \\ R. C. GUNNING
}

0. Introduction. When a group $\mathfrak{5}$ acts as a group of complex analytic automorphisms on a complex analytic manifold $\mathfrak{H}$, the group $\mathfrak{G}$ can also be considered as acting as a group of linear transformations on the space $\mathscr{A}$ of holomorphic functions on $\mathfrak{H}$, by translation of the argument; the action of an element $M \in \mathfrak{G}$ on a holomorphic function $f(z) \in \mathscr{A}$ is defined to yield the function

$$
(f \mid M)(z)=f(M z) \in \mathscr{A} .
$$

More generally, whenever there is a factor of automorphy $\rho(M, z)$ for the action of $\mathfrak{F}$ on $\mathfrak{H}$ (that is, a function on $\mathfrak{F} \times \mathfrak{H}$ which is holomorphic on $\mathfrak{H}$ and which satisfies a functional equation analogous to the chain rule for Jacobians, $[4 ; 5]$ ), it is possible to define another action of $\mathfrak{B}$ on $\mathscr{A}$ by putting

$$
\left(\left.f\right|_{\rho} M\right)(z)=\rho(M, z)^{-1} f(M z) .
$$

The invariant elements of $\mathscr{A}$, under this group action, are the automorphic or modular forms associated to the factor of automorphy $\rho$ for $\mathfrak{G}$, as in $[4 ; 5]$. To different factors of automorphy $\rho, \hat{\rho}$ there correspond of course different actions of $\mathfrak{G}$ on $\mathscr{A}$, and thus different families of automorphic forms. There then arises the question of the extent to which these group actions on families of automorphic forms are really distinct. More precisely, one can ask whether there are any linear mappings $\mathscr{D}: \mathscr{A} \rightarrow \mathscr{A}$ which commute with these group operations, in the sense that $\mathscr{D}\left(\left.f\right|_{\rho} M\right)=\left.(\mathscr{D} f)\right|_{\hat{\rho}} M$ for all $M \in \mathbb{G}$ and $f \in \mathscr{A}$. When the two factors of automorphy are equivalent, in the sense which arises naturally in the classification problem [4], there are trivial such isomorphisms $\mathscr{D}$. However, more interestingly, there are cases in which the factors $\rho, \hat{\rho}$ are inequivalent, but in which there are such homomorphisms $\mathscr{D}$. In these cases, the resulting maps can lead to quite interesting and nontrivial relations between various classes of automorphic forms. For one-dimensional complex manifolds $\mathfrak{H}$, this problem was discussed in [5]; the homomorphisms there led in a natural way to the relations between the Eichler cohomology groups and automorphic forms. In the present paper I shall discuss and classify the linear differential operators which provide such homomorphisms, when $\mathfrak{G}$ is the symplectic group or a general subgroup thereof and $\mathfrak{S}$ is the Siegel generalized upper half-plane.

Received by the editors April 19, 1962. 
In more detail, the plan of this paper is as follows. The first section contains the basic definitions and establishes the notation to be used in the sequel. It is almost necessary to consider from the outset families of holomorphic functions whose values lie in a general finite-dimensional complex vector space, and correspondingly, matrix-valued factors of automorphy. Futhermore, it is more convenient to consider, as a preliminary, the subgroup $\mathfrak{F}_{\infty} \subset \mathfrak{F}$ consisting of those symplectic matrices which act linearly on the Siegel space $\mathfrak{H}$. The second section is devoted to the determination of those differential operators commuting with the actions of the subgroup $\mathfrak{F}_{\infty}$; these are called $\mathfrak{G}_{\infty}$-homomorphisms, and the results are collected in Theorem 1. As an illustration, and to provide the detailed knowledge of these operators which will be needed for later applications, all such operators of first and second orders, in the Siegel space of rank two, are determined explicitly in the third section; the principal results are to be found in Corollaries 1.1 and 1.2, in that section. Returning to the general case, the fourth section is devoted to the determination of the differential operators which actually commute with the actions of the full group $(5$. The general criterion for such operators is given in Theorem 2; more detailed and explicit versions of the criterion, particularly for first and second order differential operators, are given in the corollaries to Theorem 2, and especially in Corollaries 2.5 and 2.6. These results are then applied, in the fifth section, to give the complete list of first and second order operators commuting with the actions of $\mathfrak{b}$ on the Siegel space of rank two; the principal results are collected in Theorem 3. Some simple applications, as illustrations of further applications which will be discussed elsewhere, are also given in the fifth section.

The selection of the symplectic group acting on the Siegel generalized upper half-plane for a detailed investigation here was, of course, motivated by the applications of the automorphic forms in this case to problems in analytic number theory, in particular to the analytic theory of quadratic forms; see $[10 ; 11]$. It is perhaps not out of place to discuss briefly in this introduction, the general structure of which the rest of this paper treats a special case, albeit a special case of independent interest; an equally detailed discussion of the general situation appears unjustified at present, because of lack of applications. In place of a factor of automorphy, one can consider its localization: for a discrete group $\mathfrak{G}$, this is a complex vector bundle over the quotient space $\mathfrak{S} /(\mathfrak{5}$; and for a transitive group, this is a homogeneous vector bundle over $\mathfrak{H}$. The general problem posed is that of determining bundle morphisms, in particular those defined in terms of differential operators. For example, when considering the tangent bundle to the manifold $\mathfrak{H}$, or general tensor bundles over $\mathfrak{H}$, there are the familiar operations of covariant derivation; these operators, however, are not generally complex analytic, although they reduce to complex analytic operators (the usual exterior derivatives) when restricted to the appropriate bundles of skew-symmetric tensors. There is, however, a further and yet more interesting aspect of the 
problem. In the special case of the symplectic group acting on the Siegel space, the coordinate transformations have a very special form. The analogue of this in the general case is the restriction to a subclass of coordinate systems on the manifold $\mathfrak{H}$, so that the coordinate transformations lie in some specified infinite Lie pseudogroup of transformations [3]; that is to say, the manifolds $\mathfrak{S}$ are restricted so as to have a pseudogroup structure still finer than just the complex analytic structure. Since this sort of restriction reduces the differentiation operators to special classes, there are in general more bundle morphisms which can then be introduced. In the case of one complex variable, this approach is really in the form given by Bol in [2], before Eichler noticed the applications to modular forms.

\section{Definitions and notation.}

(1) Throughout this discussion the usual matrix notations and conventions will be used freely. Thus for any matrix $M$, the inverse matrix will be denoted by $M^{-1}$, the transposed matrix will be denoted by ${ }^{t} M$, and the determinant of the matrix $M$ will be denoted by $\operatorname{det} M$. As a further notational convenience, the entries in the matrix $M$ will be denoted by $M_{i j}$ or $m_{i j}$; here $M_{i j}$ is the entry in the $i$ th row and $j$ th column of $M$. The entries in the matrix $M^{-1}$ will be denoted correspondingly by $M_{i j}^{-1}$, and the entries of ${ }^{t} M$ by ${ }^{t} M_{i j}$; hence ${ }^{t} M_{i j}=M_{j i}$. Unless otherwise specified, the matrix indices $i, j, k, l, \cdots$ will have the range $1, \cdots, n$ for a fixed but generally arbitrary integer $n$; and the indication of this range will be omitted in summation symbols or other formulas where no confusion can result thereby.

The Siegel generalized upper half-plane of rank $n$ is the set $\mathfrak{H}$ consisting of those $n \times n$ complex symmetric matrices $Z=X+i Y$ such that $Y=\operatorname{Im} Z$ is positive definite. The set $\mathfrak{H}$ is then a complex manifold, indeed an open subset of the space of $\frac{1}{2} n(n+1)$ complex variables $Z_{i j}=z_{i j}$, $(i \leqq j)$; of greater interest though is the fact that $\mathfrak{H}$ is a homogeneous complex manifold, under the action of the symplectic group $(5=\operatorname{Sp}(n, \mathbb{R})$ of rank $n$. Recall that the symplectic group is the subgroup $(\mathfrak{F}=\operatorname{Sp}(n, \mathbb{R}) \subset \mathrm{GL}(2 n, \mathbb{R})$ consisting of those $2 n \times 2 n$ matrices $M \in \mathrm{GL}(2 n, \mathrm{R})$ such that ${ }^{t} M J M=J$, where $J=\left(\begin{array}{c}0 I \\ -10\end{array}\right), I=I_{n}$ is the $n \times n$ identity matrix, and $0=0_{n}$ is the $n \times n$ zero matrix; if a matrix $M \in \mathrm{GL}(2 n, \mathbb{R})$ is decomposed into $n \times n$ matrix blocks $M=\left(\begin{array}{c}A \\ C\end{array}\right)$, then the condition that $M$ be symplectic has the form:

$$
{ }^{t} A C={ }^{t} C A, \quad{ }^{t} B D={ }^{t} D B, \quad{ }^{t} A D-{ }^{t} C B=I .
$$

Such a symplectic matrix $M \in \mathbb{G}$ determines a complex analytic automorphism of the generalized upper half-plane of the form:

$$
M: Z \rightarrow M(Z)=(A Z+B)(C Z+D)^{-1} .
$$

The group $\mathfrak{G}$ is then a transitive group of transformations of the space $\mathfrak{G}$. The 
isotropy subgroup of $\mathfrak{F}$ at the point $i I \in \mathfrak{H}$ is the group $\mathfrak{R}=\{M \in \mathfrak{G} \mid M(i I)=i I\}$; it follows readily that $\mathfrak{R}$ consists of those elements $M=\left(\begin{array}{c}D \\ C\end{array}-C\right) \in \mathfrak{D}$ such that $C i+D$ is a unitary matrix, hence, that $\mathcal{R}$ is canonically isomorphic to the unitary group of rank $n$. A more detailed discussion of these results can be found in the fundamental papers of Siegel $[10 ; 11]$.

A subgroup of the symplectic group which will play an important role in the subsequent discussion is the subgroup $\mathfrak{F}_{\infty} \subset \mathfrak{F}$ consisting of those symplectic matrices with $C=0$; it follows directly from the conditions (1.1) that this subgroup can be characterized as follows:

$$
\mathfrak{G}_{\infty}=\left\{M=\left(\begin{array}{rr}
{ }^{t} C & S C^{-1} \\
0 & C^{-1}
\end{array}\right) \in \mathrm{GL}(2 n, \mathbb{R}) \mid S \text { symmetric and } C \text { nonsingular }\right\}
$$

When acting on the generalized upper half-plane $\mathfrak{H}$ a matrix $M \in \mathfrak{G}_{\infty}$ of the form (1.3) has the effect:

$$
M: Z \rightarrow M(Z)={ }^{t} C Z C+S
$$

thus $\mathfrak{G}_{\infty}$ consists precisely of those symplectic transformations which act linearly on the generalized upper half-plane. It is quite easy to verify that the group $\mathfrak{b}_{\infty}$ is also transitive on the half-plane $\mathfrak{H}$, yielding another representation of $\mathfrak{H}$ as a complex homogeneous space. Actually, the space $\mathfrak{H}$ is a tube over a domain of positivity, or generalized half-plane, as introduced by Koecher; and $\mathbf{G}_{\infty}$ is the linear group under which $\mathfrak{H}$ is homogenous. Much of the discussion here can be carried over quite directly to the more general situation; however, the details vary considerably from case to case, and since it is just these details which are the most interesting for the applications, only the Siegel half-plane will be treated here. Further general discussions of domains of positivity and related matters can be found in the work of Koecher [7] and Rothaus [9].

The subgroup $\mathfrak{G}_{\infty}$ in turn contains two further subgroups, which it is convenient to consider separately. The first of these is the subgoup $\mathfrak{I} \subset \mathfrak{F}_{\infty}$ defined as:

$$
\mathfrak{I}=\left\{M=\left(\begin{array}{cc}
I & S \\
0 & I
\end{array}\right) \in \operatorname{GL}(2 n, \mathbb{R}) \mid S \text { symmetric }\right\}
$$

the elements of $\mathfrak{I}$ will be called translations for short, since their action on the generalized upper half-plane $\mathfrak{H}$ has the form $M(Z)=Z+S$. The other is the subgroup $\mathfrak{R} \subset \mathfrak{G}_{\infty}$ defined as:

$$
\Re=\left\{M=\left(\begin{array}{ll}
{ }^{t} C & 0 \\
0 & C^{-1}
\end{array}\right) \in \mathrm{GL}(2 n, \mathbb{R}) \mid C \text { nonsingular }\right\} ;
$$

the elements of $\Re$ will be called, rather incorrectly, rotations, and their action on the half-plane $\mathfrak{H}$ is of the form $M(Z)={ }^{t} C Z C$. It is an immediate consequence 
of these definitions that every element $M \in \mathfrak{G}_{\infty}$ can be written as the product of a rotation and a translation. A similar result holds for the full group $\mathfrak{6}$, except that it is necessary to consider also the involution $J=\left(\begin{array}{rr}0 & I \\ -1 & 0\end{array}\right) \in \mathbb{G}$; more precisely:

LEMMA 1. Every element $M \in \mathbb{5}$ can be written as a finite product of rotations translations, and involutions.

Proof. Consider any element $M=\left(\begin{array}{cc}\boldsymbol{A} & \boldsymbol{B} \\ C & D\end{array}\right) \in \mathbb{G}$. There is a real symmetric matrix $S$ such that $C S+D$ is a nonsingular matrix; for if det $(C S+D)=0$ for all real symmetric $S$, then necessarily $\operatorname{det}(C Z+D)=0$ for all complex symmetric $Z$, contradicting the well-known fact that $\operatorname{det}(C Z+D) \neq 0$ for $Z \in \mathfrak{H}[10]$. Then the matrix $\left(\begin{array}{ll}I & S \\ 0 & I\end{array}\right)$ is a translation, and is such that $M_{1}=M \cdot\left(\begin{array}{l}I \\ 0\end{array}\right)=\left(\begin{array}{ll}A & B_{1} \\ C & D_{1}\end{array}\right)$ where $D_{1}$ is nonsingular; and hence

is a rotation and is such that

$$
\left(\begin{array}{cc}
D_{1} & 0 \\
0 & D_{1}^{-1}
\end{array}\right)
$$

$$
M_{2}=M_{1}\left(\begin{array}{ll}
{ }^{t} D_{1} & 0 \\
0 & D_{1}^{-1}
\end{array}\right)=\left(\begin{array}{ll}
A_{2} & B_{2} \\
C_{2} & I
\end{array}\right) .
$$

Since $M_{2} \in \mathbb{G}$, it follows from (1.1) that $B_{2}$ is a symmetric matrix, and so

$$
\left(\begin{array}{cc}
I & -B_{2} \\
0 & I
\end{array}\right) M_{2}=\left(\begin{array}{ll}
A_{3} & 0 \\
C_{2} & I
\end{array}\right)=M_{3} \text {. }
$$

Again, by recalling (1.1), it follows that $A_{3}=I$, hence, $M_{3}=\left(\begin{array}{ll}1 & 0 \\ c_{2} & I\end{array}\right)$. Finally, using the involution $J \in \mathfrak{G}$, observe that $J M_{3} J^{3}=J M_{3}(-J)=\left(\begin{array}{cc}I-C_{2} \\ 0 & I\end{array}\right) \in(5)$ is a translation, and the lemma is thereby demonstrated.

(2) Let $\rho$ be a holomorphic, linear representation of the general linear group $\mathrm{GL}(n, \mathbb{C})$, with representation space $V_{\rho}$; that is to say, let $\rho$ be a complex analytic homomorphism of $\mathrm{GL}(n, \mathbb{C})$ into the complex Lie group of automorphisms of the finite-dimensional complex vector space $V_{\rho}$. To each symplectic matrix $M=\left(\begin{array}{cc}A & B \\ C & D\end{array}\right)$ associate the function

$$
\rho(M, Z)=\rho(C Z+D),
$$

which is holomorphic on $\mathfrak{S}$ and has values in the group of automorphisms of $V_{\boldsymbol{\rho}}$. These functions determine a factor of automorphy for the group $\mathbb{6}$ acting on the space $\mathfrak{H}$, as discussed in [4] or [5], for example; for any two symplectic matrices $M, N \in \mathfrak{G}$, the factors of automorphy satisfy the functional equations $\rho(M N, Z)=\rho(M, N Z) \rho(N, Z)$. Any such factor of automorphy in turn leads to a representation of the symplectic group $(5$ as a group of linear transformations on the complex vector space $\Phi_{\rho}=\Phi\left(\mathfrak{H}, V_{\rho}\right)$ of $C_{\infty}$ mappings of the space $\mathfrak{H}$ into the vector space $V_{\rho}$, as in [5]. This representation is that which associates to any function $F \in \Phi_{\rho}$ and any symplectic matrix $M \in \mathfrak{G}$ the function $\left.F\right|_{\rho} M \in \Phi_{\rho}$ defined by: 


$$
\left(\left.F\right|_{\rho} M\right)(Z)=\rho(M, Z)^{-1} F(M Z) .
$$

Thus $\Phi_{\rho}$ has not only the structure of a complex vector space, but also the structure of a $\mathfrak{G}$-module, with the action of $\mathfrak{G}$ as in (1.8); to emphasize the fact that both of these structures are to be considered, $\Phi_{\rho}$ will be called a $(5$-space. Note that for any subgroup of $\mathfrak{G}$, such as $\mathfrak{G}_{\infty}$, the same vector space $\Phi_{\rho}$ can be considered as a $\mathfrak{G}_{\infty}$-space as well; this is a weaker structure, in the obvious sense of the phrase. Note, further, that any subspace of $\Phi_{\rho}$ which is preserved under the action of $\mathfrak{G}$ can also be considered as a $\mathfrak{W}$-space, or as a $\mathfrak{G}_{\infty}$-space. The subspace which is of particular interest from the beginning is, of course, the space $\Theta_{\rho}=\Theta\left(\mathfrak{H}, V_{\rho}\right)$ of holomorphic mappings of $\mathfrak{H}$ into $V_{\rho}$.

Now consider two representations $\rho, \hat{\rho}$ of $\operatorname{GL}(n, \mathbb{C})$, with representation spaces $V_{\rho}, V_{\hat{\rho}}$ and $\Phi_{\rho}, \Phi_{\hat{\rho}}$, respectively, as defined just above. For any function $F \in \Phi_{\rho}$, the various partial derivatives $\partial^{\mu} F(z) / \partial z_{i_{1} j_{1}} \cdots \partial z_{i \mu j \mu}$ are functions belonging to the same space $\Phi_{\rho}$; for $C^{\infty}$ functions, the complex differential operator is defined to be the first order linear differential operator $2 \partial / \partial z_{i j}=\partial / \partial x_{i j}-\sqrt{ }-1 \partial / \partial y_{i j}$, where $z_{i j}=x_{i j}+\sqrt{ }-1 y_{i j}$. If $A_{i_{1} j_{1} \ldots i_{1} j_{n}}(Z)$ are arbitrary $C^{\infty}$ mappings from $\mathfrak{H}$ into the space of linear maps of $V_{\rho}$ into $V_{\hat{\rho}}$, then the composition

$$
(\mathscr{D} F)(Z)=\sum_{\mu=0}^{v} \sum_{i_{1} \leqq j_{1} \ldots i_{\mu} \leqq j_{\mu}} A_{i_{1} j_{1} \ldots i_{\mu} j_{\mu}}(Z) \partial^{\mu} F(Z) / \partial Z_{i_{1} j_{1}} \cdots \partial Z_{i_{\mu} j}
$$

represents a linear mapping $\mathscr{D}: \Phi_{\rho} \rightarrow \Phi_{\hat{\rho}}$, which will be called a linear differential mapping of order $v$. In case that the coefficients in (1.9) are actually holomorphic on $\mathfrak{H}$, the restriction of $\mathscr{D}$ defines a linear mapping $\mathscr{D}: \Theta_{\rho} \rightarrow \Theta_{\hat{\rho}}$. When saying that $\mathscr{D}$ is of order $v$ it will be supposed that not all the coefficients of order $v$ in (1.9) vanish.

A general holomorphic mapping $\mathscr{D}: \Theta_{\rho} \rightarrow \Theta_{\hat{\rho}}$ of the form (1.9), with no condition imposed on the coefficients except that they be holomorphic on $\mathfrak{H}$, is a homomorphism when $\Theta_{\rho}$ and $\Theta_{\hat{\rho}}$ are considered merely as linear spaces. The question then arises, when such a mapping is, in addition, a homomorphism between these two spaces when they are considered as (5-spaces; mappings of the form (1.9) with these additional properties will be called holomorphic differential (5-homomorphisms, of order $v$. The condition that the mapping (1.9) be a (5-homomorphism is, of course, simply that

$$
\mathscr{D}\left(\left.F\right|_{\rho} M\right)=\left.(\mathscr{D} F)\right|_{\hat{\rho}} M
$$

for all $F \in \Phi_{\rho}$ and all $M \in(5$. The present paper is devoted to the problem of determining more explicitly all the holomorphic differential 5 -homomorphisms. These are mappings which arise in some investigations of automorphic functions on the space $\mathfrak{G}$, as indicated briefly in [5]; indeed, the purpose of this classification is for such applications. In approaching this problem it is convenient first to 
determine the larger class of holomorphic differential $\mathfrak{b}_{\infty}$-homomorphisms, and

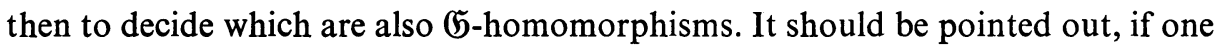
is interested in $C^{\infty}$ rather than just in holomorphic functions, that there are many differential $\mathfrak{5}$-homomorphisms $\mathscr{D}: \Phi_{\rho} \rightarrow \Phi_{\hat{\rho}}$ which are not holomorphic, and, hence, which do not correspond to holomorphic $G$-homomorphisms $\mathscr{D}: \Theta_{\rho} \rightarrow \Theta_{\hat{\rho}}$; these will not appear in the present classification, therefore. Examples of such mappings have been given in $[5 ; 6]$.

\section{Holomorphic differential $\mathfrak{G}_{\infty}$-homomorphisms.}

(3) For the purpose of this classification it is convenient to introduce a suggestive and, eventually, simpler notation for the differential operators (1.9). For any integer $\mu=0,1,2, \cdots$ consider the finite dimensional complex vector space of tensors $\xi_{i_{1} j_{1} \ldots i_{\mu} j_{\mu}}$, in the sense of Weyl [12]; in another terminology, this space is the tensor product of the complex vector space $\mathbb{C}^{n}$ with itself $2 \mu$ times. Let $V_{\mu}$ be the subspace consisting of those tensors with the following symmetries:

$$
\begin{aligned}
& \xi_{i_{1} j_{1} \ldots i, j_{2} \ldots i j_{j}}=\xi_{i_{1} j_{1} \ldots j_{\nu}, \ldots \ldots i j .,}, \quad \text { any } \alpha \text {; } \\
& \xi_{i_{1} j_{1} \ldots i_{\alpha} j_{\alpha} \ldots i_{\beta} j_{\beta} \ldots i_{j} j_{.,}}=\xi_{i_{1} j_{1} \ldots i_{\beta} j_{\beta} \ldots i_{\alpha} j_{\alpha} \ldots i_{\mu} j_{\mu}}, \quad \text { any } \alpha, \beta \text {. }
\end{aligned}
$$

To any such tensor $\xi \in V_{\mu}$ and to any matrix $C=\left(c_{i j}\right) \in \operatorname{GL}(n, \mathbb{C})$ associate the tensor $\sigma_{\mu}(C) \xi$ which has coefficients

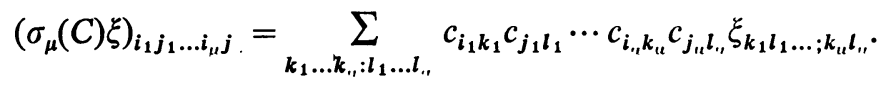

It is clear that $\sigma_{\mu}(C) \xi \in V_{\mu}$, and that the mapping $\xi \rightarrow \sigma_{\mu}(C) \xi$ is a linear transformation $\sigma_{\mu}(C)$ on the vector space $V_{\mu}$; indeed, the mapping $C \rightarrow \sigma_{\mu}(C)$ is a holomorphic linear representation of $\mathrm{GL}(n, \mathbb{C})$, with the representation space $V_{\mu}$. It should be remarked here that the representation $\sigma_{\mu}$ is generally not irreducible; a detailed discussion of the decomposition of this representation in a special case will appear in the following section (Lemma 5).

Now consider an arbitrary function $F \in \Phi_{\rho}$, which is thus a $C^{\infty}$ mapping $F: \mathfrak{H} \rightarrow V_{\rho}$; upon choosing a basis for the vector space $V_{\rho}$, the function $F$ can be described by its components $F(Z)=\left\{f_{a}(Z)\right\}$. For each fixed point $Z \in \mathfrak{H}$ the set of partial derivatives $\left\{\partial^{\mu} f_{\alpha}(Z) / \partial z_{i_{1} j_{1}} \cdots \partial z_{i, j . j}\right\}$ can be envisaged as forming a tensor $\partial_{\mu} F(Z) \in V_{\rho} \otimes V_{\mu}$, since the symmetry conditions (2.1) and (2.2) are clear; the tensor $\partial_{\mu} F(Z)$, that is to say, is defined to have the components

$$
\left(\partial_{\mu} F(Z)\right)_{\alpha i_{1} j_{1} \ldots i j_{j . .}}=\partial^{\mu} f_{\alpha}(Z) / \partial z_{i_{1_{j} j_{1}}} \cdots \partial z_{i_{j} .} .
$$

The mapping $Z \rightarrow \partial_{\mu} F(Z)$ is then a $C^{\infty}$ mapping $\partial_{\mu} F: \mathfrak{T} \rightarrow V_{\rho} \otimes V_{\mu}$; and if $F \in \Theta_{\rho}$, the mapping $\partial_{\mu} F$ is by the same light a holomorphic mapping. Suppose further that for each fixed $Z \in \mathfrak{S}$ there is given a linear mapping $\mathscr{A}_{\mu}(Z): V_{\rho} \otimes V_{\mu} \rightarrow V_{\hat{\rho}}$, such that the mapping $Z \rightarrow \mathscr{A}_{\mu}(Z)$ is actually a holomorphic mapping from $\mathfrak{Y}$ to 
the space of maps $V_{\rho} \otimes V_{\mu} \rightarrow V_{\hat{\rho}}$. Then there is a linear mapping $\mathscr{D}: \Theta_{\rho} \rightarrow \Theta_{\hat{\rho}}$ defined by

$$
\mathscr{D} F=\sum_{\mu=0}^{\nu} \mathscr{A}_{\mu}(Z) \partial_{\mu} F(Z)
$$

If a basis is selected for the space $V_{\hat{\rho}}$ as well, the linear transformation $\mathscr{A}_{\mu}(Z)$ is represented by a matrix with coefficients $A_{\beta \alpha i_{1} j_{1} \ldots i_{\mu j}}(Z)$; and the linear operator (2.5) then takes the form (1.9). Thus the mapping (2.5) is a holomorphic linear differential mapping of order $v$. Conversely, it is clear that the coefficients in (1.9) can, without loss of generality, be taken to satisfy the symmetry conditions (2.1) and (2.2); so every holomorphic linear differential mapping can be put into the form (2.5).

(4) The differential operator (2.5) is now a holomorphic $\mathfrak{( 5}_{\infty}$-homomorphism if and only if its coefficients $\mathscr{A}_{\mu}(Z)$ are holomorphic, and it satisfies (1.10) for all $F \in \Theta_{\rho}$ and all $M \in \mathfrak{G}_{\infty}$. However, since every $M \in \mathfrak{G}_{\infty}$ is the product of a translation (1.5) and a rotation (1.6), it suffices to impose condition (1.10) in these two cases separately.

LEMMA 2. A holomorphic linear differential mapping (2.5) satisfies (1.10) for all $F \in \Theta_{\rho}$ and all translations $M \in \mathfrak{I}$ if and only if the coefficients are constants.

Proof. Consider a translation $M$ of the form (1.5), and put $W=M(Z)=Z+S$. It is clear from (1.5) and (1.7) that $\rho(M, Z)=I$ and that $\hat{\rho}(M, Z)=I$; and it is likewise clear that $\partial_{\mu}\left(\left(\left.F\right|_{\rho} M\right)(Z)\right)=\left(\partial_{\mu} F\right)(W)$. Thus condition $(1.10)$ takes the form

$$
\sum_{\mu=0}^{v} \mathscr{A}_{\mu}(Z) \partial_{\mu} F(W)=\sum_{\mu=0}^{\nu} \mathscr{A}_{\mu}(W) \partial_{\mu} F(W)
$$

Since (2.6) is required to hold for all $F \in \Theta_{\rho}$, it follows that

$$
\mathscr{A}_{\mu}(Z)=\mathscr{A}_{\mu}(W)=\mathscr{A}_{\mu}(Z+S)
$$

for all real symmetric matrices $S$; but since the coefficients $\mathscr{A}_{\mu}(Z)$ are also required to be holomorphic, it follows that they must indeed be constants, as desired.

REMARK. It is at just this point that the doors are opened to admit a much larger class of operators if the coefficients are not required to be holomorphic; see [6] in this connection.

LEMMA 3. A linear differential operator of the form (2.5) satisfies (1.10) for all $F \in \Theta_{\rho}$ and all rotations $M \in \Re$ if and only if (as linear mappings on $\left.V_{\rho} \otimes V_{\mu}\right)$ 


$$
\hat{\rho}(C) \cdot \mathscr{A}_{\mu}=\mathscr{A}_{\mu} \cdot\left[\rho(C) \otimes \sigma_{\mu}(C)\right]
$$

for all $C \in \mathrm{GL}(n, \mathbb{C})$ and for $\mu=1,2, \cdots, v$.

Proof. Consider a rotation $M \in \mathfrak{R}$, of the form (1.6) for an arbitrary $C \in \mathrm{GL}(n, \mathbb{R})$; and put $W=M(Z)={ }^{t} C Z C$. It is clear from (1.6) and (1.7) that $\rho(M, Z)=\rho\left(C^{-1}\right)=\rho(C)^{-1}$ and that $\hat{\rho}(M, Z)=\hat{\rho}\left(C^{-1}\right)=\hat{\rho}(C)^{-1}$; thus, for instance, $\left(\left.F\right|_{\rho} M\right)(Z)=\rho(C) F(W)$. Now note that, in terms of the components which serve as complex coordinates on the space $\mathfrak{H}$, the transformation $M$ can be written:

$$
w_{i j}=\sum_{k, l} z_{k l} c_{k i} c_{l j}=\sum_{k \leqq l}\left(1-\frac{1}{2} \delta_{l}^{k}\right) z_{k l}\left(c_{k i} c_{l j}+c_{k j} c_{l i}\right),
$$

where $\delta_{l}^{k}$ is the familiar Kronecker symbol; therefore,

$$
\frac{\partial w_{i j}}{\partial z_{k l}}=\left(1-\frac{1}{2} \delta_{l}^{k}\right)\left(c_{k i} c_{l j}+c_{k j} c_{l i}\right),
$$

a constant independent of $Z \in \mathfrak{H}$. Therefore,

$$
\begin{aligned}
\partial_{\mu}(F \mid \rho)(Z) & =\left\{\rho(C) \partial^{\mu} F(W) / \partial z_{k_{1} l_{1}} \cdots \partial z_{k_{\mu} l_{\mu}}\right\} \\
& =\left\{\rho(C) \sum_{i \leqq j} \frac{\partial w_{i_{1} j_{1}}}{\partial z_{k_{1} l_{1}}} \cdots \frac{\partial w_{i_{\mu} j_{\mu}}}{\partial z_{k_{\mu} l_{\mu}}} \partial^{\mu} F(W) / \partial w_{i_{1} j_{1}} \cdots \partial w_{i_{\mu} j_{\mu}}\right\} \\
& =\left\{\rho(C) \sum_{i, j} c_{k_{1} i_{1}} c_{l_{1} j_{1}} \cdots c_{k_{\mu} i_{\mu}} c_{l_{\mu} j_{\mu}} \partial^{\mu} F(W) / \partial w_{i_{1} j_{1}} \cdots \partial w_{i_{\mu} j_{\mu}}\right\} \\
& =\left[\rho(C) \otimes \sigma_{\mu}(C)\right] \cdot \partial_{\mu} F(W) .
\end{aligned}
$$

Now condition (1.10) takes the form

$$
\sum_{\mu=0}^{v} \mathscr{A}_{\mu}(Z) \cdot\left[\rho(C) \otimes \sigma_{\mu}(C)\right] \cdot \partial_{\mu} F(W)=\hat{\rho}(C) \sum_{\mu=0}^{v} \mathscr{A}_{\mu}(W) \cdot \partial_{\mu} F(W) ;
$$

but since the condition (2.8) is required to hold for all $F \in \Theta_{\rho}$, it follows that

$$
\mathscr{A}_{\mu}(Z) \cdot\left[\rho(C) \otimes \sigma_{\mu}(C)\right]=\hat{\rho}(C) \cdot \mathscr{A}_{\mu}(W)
$$

for each $\mu$ individually. This must now hold for all $C \in \mathrm{GL}(n, \mathrm{R})$; however, since both sides are holomorphic functions of the matrix $C$, the same result actually holds for all $C \in \mathrm{GL}(n, \mathbb{C})$, thus completing the proof.

It is a familiar fact, as discussed in [12] for instance, that the tensor product of two representations of the general linear group can be decomposed into a direct sum of irreducible components; the notation $\hat{\rho} \subset \rho \otimes \sigma$ will be used to indicate that the irreducible components of the representation $\hat{\rho}$, counting multiplicities occur among the components of the decomposition of the representation $\rho \otimes \sigma$. 
THEOREM 1. If $\rho$ and $\hat{\rho}$ are irreducible holomorphic representations of $\mathrm{GL}(n, \mathbb{C})$, then there exists a holomorphic differential $\mathfrak{G}_{\infty}$-homomorphism $\mathscr{D}: \Theta_{\rho} \rightarrow \Theta_{\hat{\rho}}$ of order $v$ if and only if

$$
\hat{\rho} \subset \rho \otimes \sigma_{v} ;
$$

all coefficients $\mathscr{A}_{\mu}(Z)$ of $\mathscr{D}$ are zero except the leading coefficient $\mathscr{A}_{v}(Z)$, which is a constant linear mapping such that

$$
\hat{\rho}(C) \cdot \mathscr{A}_{v}=\mathscr{A}_{v} \cdot\left[\rho(C) \otimes \sigma_{v}(C)\right]
$$

for all $C \in \mathrm{GL}(n, \mathbb{C})$.

Proof. It follows immediately from Lemmas 2 and 3 that there exists a holomorphic differential $\mathfrak{G}_{\infty}$-homomorphism $\mathscr{D}: \Theta_{\rho} \rightarrow \Theta_{\hat{\rho}}$ if and only if there are constant linear mappings $\mathscr{A}_{\mu}, \mu=0,1, \cdots, v$, such that $\mathscr{A}_{\mu} \cdot\left[\rho(C) \otimes \sigma_{\mu}(C)\right]=\hat{\rho}(C) \cdot \mathscr{A}_{\mu}$ for all $C \in \mathrm{GL}(n, \mathbb{C})$. Schur's lemma shows that if $\mathscr{A}_{\mu} \neq 0$ then $\hat{\rho} \subset \rho \otimes \sigma_{\mu}$; conversely, if $\hat{\rho} \subset \rho \otimes \sigma_{\mu}$, then there obviously exists a nonzero linear transformation $\mathscr{A}_{\mu}$ with the above property. The representations $\hat{\rho}$ and $\rho$, being irreducible are homogeneous rational functions of some fixed degrees $[1 ; 12]$; and since $\sigma_{\mu}$ is a homogeneous rational function of degree $2 \mu$, it is clear that $\hat{\rho} \subset \rho \otimes \sigma_{\mu}$ for at most one value of $\mu$. The desired theorem is an obvious consequence of these observations.

REMARK. If the representations $\rho$ and $\hat{\rho}$ are not assumed to be irreducible, then (2.9) must be replaced by the condition that

$$
\hat{\rho} \subset \rho \otimes\left(\sum_{\mu=0}^{v} \sigma_{\mu}\right),
$$

and that some component of $\hat{\rho}$ is contained in $\rho \otimes \sigma_{v}$; the second part of the theorem must also be modified, in the obvious manner.

\section{Some examples of $\mathfrak{b}_{\infty}$-homomorphisms.}

(5) The preceding discussion is well illustrated by the special case $n=2$, which is also sufficiently simple that the results can be written out quite explicitly. First, however, it is necessary to interject a preliminary discussion of the group representations involved; the general properties of these representations are discussed in $[12 ; 1]$, while the notation which will be used here is that of $[6]$. For the group $\mathrm{GL}(2, \mathbb{C})$ the general irreducible holomorphic representation is of the form $\Delta^{\lambda}\{f\}$, where $\lambda$ is an arbitrary integer, and $f$ is a non-negative integer. Here $\Delta$ is the one-dimensional representation $\Delta(C)=\operatorname{det} C$, for any $C \in \mathrm{GL}(2, \mathbb{C})$; and $\{f\}$ is the $(f+1)$-dimensional representation which has the form (2.3), but where the representation space consists of fully symmetric tensors of degree $f$. To describe the character $\phi_{\rho}$ of the representation $\rho=\Delta^{\lambda}\{f\}$ it suffices to consider diagonal matrices of the form $\left(\begin{array}{c}\varepsilon \\ 0 \\ 1\end{array}\right)$ for $\varepsilon \in \mathbb{C}$; and for such a matrix, 


$$
\phi_{\rho}\left(\begin{array}{ll}
\varepsilon & 0 \\
0 & 1
\end{array}\right)=\varepsilon^{\lambda}\left(\varepsilon^{f}+\varepsilon^{f-1}+\cdots+\varepsilon+1\right) .
$$

Two rather more detailed properties of the representations of $\operatorname{GL}(2, \mathbb{C})$ are also needed for the present discussion: first is the analysis of the irreducible components of the Kronecker product of two representations; second is the analysis of the irreducible components of the representation $\sigma_{v}$.

LEMMA 4. For representations of the group $\mathrm{GL}(2, \mathbb{C})$, with $f \geqq g$ :

$$
\Delta^{\lambda}\{f\} \otimes \Delta^{\mu}\{g\}=\Delta^{\lambda+\mu}\{f+g\}+\Delta^{\lambda+\mu+1}\{f+g-2\}+\cdots+\Delta^{\lambda+\mu+g}\{f-g\} .
$$

Proof. This is rather a standard result, and can be found in [1] or in [8]; however, for completeness, and since it is so easy, another proof will be included here. It is clearly enough to consider merely the representations $\rho=\{f\}$ and $\sigma=\{g\}$, where, say, $f \geqq g$. Then for the characters it follows that, from (3.1)

$$
\begin{aligned}
& \phi_{\rho \otimes \sigma}\left(\begin{array}{ll}
\varepsilon & 0 \\
0 & 1
\end{array}\right)=\phi_{\rho}\left(\begin{array}{ll}
\varepsilon & 0 \\
0 & 1
\end{array}\right) \cdot \phi_{\sigma}\left(\begin{array}{ll}
\varepsilon & 0 \\
0 & 1
\end{array}\right)=\left(\varepsilon^{f}+\cdots+1\right) \cdot\left(\varepsilon^{g}+\cdots+1\right) \\
& =\left(\varepsilon^{f+g}+\cdots+1\right)+\left(\varepsilon^{f+g-1}+\cdots+\varepsilon\right)+\cdots+\left(\varepsilon^{f}+\cdots+\varepsilon^{g}\right)
\end{aligned}
$$

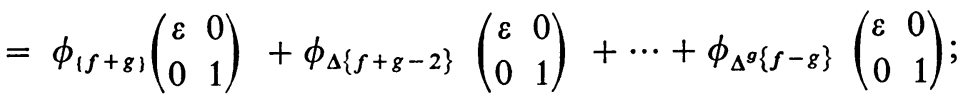

and this in turn establishes formula (3.2), as desired.

Lemma 5. For the group $\mathrm{GL}(2, \mathbb{C})$,

$$
\sigma_{v}=\{2 v\}+\Delta^{2}\{2 v-4\}+\Delta^{4}\{2 v-8\}+\cdots
$$

Proof. The representation space $V_{v}$ for the representation $\sigma_{v}$ consists of those tensors $\xi=\left\{\xi_{i_{1} j_{1} \ldots i_{v} j_{v}}\right\}$ with the symmetries (2.1) and (2.2), where $1 \leqq i, j \leqq 2$. For any non-negative integers $a, b$, with $a+b+c=v$, let $\xi(a, b, c) \in V_{v}$ be that tensor with the following components: $\xi(a, b, c)_{i_{1} j_{1} \ldots i_{v} j_{v}}=1$ if $a$ of the index pairs $\left(i_{\alpha} j_{\alpha}\right)$ have the values $(1,1), b$ of the index pairs have the values $(1,2)$ or $(2,1)$, and $c$ of the index pairs have the values $(2,2)$; and all other components are zero. In view of the symmetry conditions on $V_{v}$ it is clear that the tensors $\xi(a, b, c)$ form a basis for $V_{v}$; hence $\sigma_{v}$ is an integral rational representation of $\operatorname{GL}(2, \mathbb{C})$ of degree $2 v$ and of dimension $\frac{1}{2}(v+1)(v+2)$. Now consider a matrix $\left(\begin{array}{ll}\varepsilon & 0 \\ 0 & 1\end{array}\right) \in \operatorname{GL}(2, \mathbb{C})$, and note that

$$
\sigma_{v}\left(\begin{array}{ll}
\varepsilon & 0 \\
0 & 1
\end{array}\right) \cdot \xi(a, b, c)=\varepsilon^{2 a+b} \cdot \xi(a, b, c)
$$

hence the character $\phi_{v}$ of the representation $\sigma_{v}$ is determined by the fact that 


$$
\phi_{v}\left(\begin{array}{ll}
\varepsilon & 0 \\
0 & 1
\end{array}\right)=\sum_{a+b+c=v ; a, b, c \geqq 0} \varepsilon^{2 a+b}=\sum_{b=0}^{v} \sum_{a=0}^{v{ }^{\prime} b} \varepsilon^{2 a+b} .
$$

The latter sum can be rewritten by separating the summation over $b$ into odd and even terms and then regrouping, as follows:

$$
\begin{aligned}
\phi_{v}\left(\begin{array}{ll}
\varepsilon & 0 \\
0 & 1
\end{array}\right) & =\sum_{m=0}^{[v / 2]}\left\{\sum_{a=0}^{v-2 m} \varepsilon^{2 a+2 m}+\sum_{a=0}^{v-2 m-1} \varepsilon^{2 a+2 m+1}\right\} \\
& =\sum_{m=0}^{[v / 2]} \varepsilon^{2 m}\left\{\sum_{h=0}^{2 v-4 m} \varepsilon^{h}\right\} \\
& =\phi_{\{2 v\}}\left(\begin{array}{ll}
\varepsilon & 0 \\
0 & 1
\end{array}\right)+\phi_{\Delta}^{2}\{2 v-4\}\left(\begin{array}{ll}
\varepsilon & 0 \\
0 & 1
\end{array}\right)+\cdots
\end{aligned}
$$

since the representations whose characters appear in the last line above all have degree $2 v$, it follows that the representation $\sigma_{v}$ decomposes in the same way, which completes the proof of (3.3).

Now consider a representation $\rho=\Delta^{\lambda}\{f\}$ of $\operatorname{GL}(2, \mathbb{C})$. According to Theorem 1 , there will exist a holomorphic differential $\mathscr{G}_{\infty}$-homomorphism $\mathscr{D}: \Theta_{\rho} \rightarrow \Theta_{\hat{\rho}}$ of degree $v$ if and only if the irreducible representation $\hat{\rho}$ is one of the components of the tensor product $\Delta^{\lambda}\{f\} \otimes \sigma_{v}$; and, by Lemmas 4 and 5 , it follows that the representation $\hat{\rho}$ must be of the form $\Delta^{\lambda}\{f+2 v\}, \Delta^{\lambda+1}\{f+2 v-2\}$, $\Delta^{\lambda+2}\{f+2 v-4\}, \cdots$. The coefficients of the differential operator $\mathscr{D}$ must be such as to satisfy (2.10); and hence, the coefficients can be given in the form of generalized Kronecker tensors, of the appropriate symmetry types, as in [6].

(6) The differential operators of lower orders can be written out completely without much difficulty; the cases of degrees 1 and 2 will be treated here. The notation and terminology introduced in [6] will be used throughout. In particular, recall that a function $F \in \Phi_{\rho}$, where $\rho=\Delta^{\lambda}\{f\}$, is given by its components $F_{i_{1} \ldots i f}$; and since the components are fully symmetric in their indices, and the indices in turn take only the values 1 and 2, a component is described fully by giving the number $v$ of its indices which have the value 1 . Thus set $F_{v}=F_{1 \ldots 12 \ldots 2}$, where there are $v$ indices which have the values 1 and $f-v$ which have the value 2 .

COROLlaRY 1.1. For the irreducible holomorphic representation $\rho=\Delta^{\lambda}\{f\}$ of $\mathrm{GL}(2, \mathbb{C})$, there are holomorphic differential $G_{\infty}$-homomorphisms $\mathscr{D}: \Theta_{\rho} \rightarrow \Theta_{\hat{\rho}}$ of order 1 of the following forms, and no others:

$$
\begin{aligned}
& \text { for } \hat{\rho}=\Delta^{\lambda}\{f+2\}, f \geqq 0, \text { the operator } \mathscr{D}_{1} \text { given by } \\
& \qquad \begin{aligned}
\left(\mathscr{D}_{1} F\right)_{v}= & v(v-1) \partial F_{v-2} / \partial z_{11}-v(v-f-2) \partial F_{v-1} / \partial z_{12} \\
& +(v-f-1)(v-f-2) \partial F_{v} / \partial z_{22}
\end{aligned}
\end{aligned}
$$

where $0 \leqq v \leqq f+2$, and $F_{v}=0$ for $v<0$; 
(3.5) for $\hat{\rho}=\Delta^{\lambda+1}\{f\}, f \geqq 1$, the operator $\mathscr{D}_{2}$ given by

$$
\left(\mathscr{D}_{2} F\right)_{v}=-2 v \partial F_{v-1} / \partial z_{11}+(2 v-f) \partial F_{v} / \partial z_{12}-2(v-f) \partial F_{v+1} / \partial z_{22},
$$

where $0 \leqq v \leqq f$;

(3.6) for $\hat{\rho}=\Delta^{\lambda+2}\{f-2\}$, $f \geqq 2$, the operator $\mathscr{D}_{3}$ given by

$$
\left(\mathscr{D}_{3} F\right)_{v}=\partial F_{v} / \partial z_{11}-\partial F_{v+1} / \partial z_{12}+\partial F_{v+2} / \partial z_{22},
$$

where $0 \leqq v \leqq f-2$.

Proof. It follows from Theorem 1 that $\hat{\rho} \subset \rho \otimes \sigma_{1}$. However, by Lemma 5, note that $\sigma_{1}=\{2\}$; hence, by Lemma 4 ,

$$
\rho \otimes \sigma_{1}=\Delta^{\lambda}\{f+2\}+\Delta^{\lambda+1}\{f\}+\Delta^{\lambda+2}\{f-2\},
$$

where the second and third terms appear only for $f \geqq 1$ and $f \geqq 2$ respectively. The coefficients of the equations are, as noted above, symmetrized Kronecker symbols of the form of equation (1.20) in [6]; the particular symbols needed here were, moreover, listed explicitly in equations (2.4), (2.5), (2.6) of [6], and the lemma follows immediately from these observations.

COROLlaRY 1.2. For the irreducible holomorphic representation $\rho=\Delta^{\lambda}\{f\}$ of $\mathrm{GL}(2, \mathbb{C})$, there are holomorphic differential $\mathfrak{G}_{\infty}$-homomorphisms $\mathscr{D}: \Theta_{\rho} \rightarrow \Theta_{\hat{\rho}}$ of order 2 of the following forms, and no others:

(3.7) for $\hat{\rho}=\Delta^{\lambda}\{f+4\}, f \geqq 0$, the operator $\mathscr{D}_{4}$ given by

$$
\begin{aligned}
&\left(\mathscr{D}_{4} F\right)_{v}= v(v-1)(v-2)(v-3) \partial^{2} F_{v-4} / \partial z_{11}^{2} \\
&-2 v(v-1)(v-2)(v-f-4) \partial^{2} F_{v-3} / \partial z_{11} \partial z_{12} \\
&+v(v-1)(v-f-3)(v-f-4)\left[2 \partial^{2} F_{v-2} / \partial z_{11} \partial z_{22}\right. \\
&\left.+\partial^{2} F_{v-2} / \partial z_{12}^{2}\right] \\
&- 2 v(v-f-2)(v-f-3)(v-f-4) \partial^{2} F_{v-1} / \partial z_{12} \partial z_{22} \\
&+(v-f-1)(v-f-2)(v-f-3)(v-f-4) \partial^{2} F_{v} / \partial z_{22}^{2}
\end{aligned}
$$

where $0 \leqq v \leqq f+4$;

(3.8) for $\hat{\rho}=\Delta^{\lambda+1}\{f+2\}, f \geqq 1$, the operator $\mathscr{D}_{5}$ given by

$$
\begin{aligned}
\left(\mathscr{D}_{5} F\right)_{v}= & 2 v(v-1)(v-2) \partial^{2} F_{v-3} / \partial z_{11}^{2} \\
& -v(v-1)(4 v-3 f-8) \partial^{2} F_{v-2} / \partial z_{11} \partial z_{12} \\
& +v(v-f-2)(2 v-f-2)\left[2 \partial^{2} F_{v-1} / \partial z_{11} \partial z_{22}+\partial^{2} F_{v-1} / \partial z_{12}^{2}\right] \\
& -(v-f-2)(v-f-1)(4 v-f) \partial^{2} F_{v} / \partial z_{12} \partial z_{22} \\
& +2(v-f-2)(v-f-1)(v-f) \partial^{2} F_{v+1} / \partial z_{22}^{2}
\end{aligned}
$$


where $0 \leqq v \leqq f+2$;

(3.9) for $\hat{\rho}=\Delta^{\lambda+2}\{f\}$, any linear combination of the operators $\mathscr{D}_{6}$ (defined for $f \geqq 2$ ) and $\mathscr{D}_{7}$ (defined for $f \geqq 0$ ) given by:

$$
\begin{aligned}
\left(\mathscr{D}_{6} F\right)_{v}= & v(v-1) \partial^{2} F_{v-2} / \partial z_{11}^{2}-v(2 v-f-1) \partial^{2} F_{v-1} / \partial z_{11} \partial z_{12} \\
& +\left[v(v-f)+\frac{1}{6} f(f-1)\right]\left[2 \partial^{2} F_{v} / \partial z_{11} \partial z_{22}+\partial^{2} F_{v} / \partial z_{12}^{2}\right] \\
& -(v-f)(2 v-f+1) \partial^{2} F_{v+1} / \partial z_{12} \partial z_{22} \\
& +(v-f)(v-f+1) \partial^{2} F_{v+2} / \partial z_{22}^{2},
\end{aligned}
$$

and

$$
\left(\mathscr{D}_{7} F\right)_{v}=4 \partial^{2} F_{v} / \partial z_{11} \partial z_{22}-\partial^{2} F_{v} / \partial z_{12}^{2}
$$

where $0 \leqq v \leqq f$

(3.10) for $\hat{\rho}=\Delta^{\lambda+3}\{f-2\}, f \geqq 3$, the operator $\mathscr{D}_{8}$ given by

$$
\begin{aligned}
\left(\mathscr{D}_{8} F\right)_{v}= & 2 v \partial^{2} F_{v-1} / \partial z_{11}^{2}-(4 v-f+2) \partial^{2} F_{v} / \partial z_{11} \partial z_{12} \\
& +(2 v-f+2)\left[2 \partial^{2} F_{v+1} / \partial z_{11} \partial z_{22}+\partial^{2} F_{v+1} / \partial z_{12}^{2}\right] \\
& -(4 v-3 f+6) \partial^{2} F_{v+2} / \partial z_{12} \partial z_{22}+2(v-f+2) \partial^{2} F_{v+3} / \partial z_{22}^{2},
\end{aligned}
$$

where $0 \leqq \nu \leqq f-2$; and finally

(3.11) for $\hat{\rho}=\Delta^{\lambda+4}\{f-4\}, f \geqq 4$, the operator $\mathscr{D}_{9}$ given by

$$
\begin{aligned}
\left(\mathscr{D}_{9} F\right)_{v}= & \partial^{2} F_{v} / \partial z_{11}^{2}-2 \partial^{2} F_{v+1} / \partial z_{11} \partial z_{12}+2 \partial^{2} F_{v+2} / \partial z_{11} \partial z_{22} \\
& +\partial^{2} F_{v+2} / \partial z_{12}^{2}-2 \partial^{2} F_{v+3} / \partial z_{12} \partial z_{22}+\partial^{2} F_{v+4} / \partial z_{22}^{2},
\end{aligned}
$$

where $0 \leqq v \leqq f-4$.

Proof. It follows from Theorem 1 that $\hat{\rho} \subset \rho \otimes \sigma_{2}$, and from Lemma 5 that $\sigma_{2}=\{4\}+\Delta^{2}$; hence, by Lemma 4 ,

$$
\rho \otimes \sigma_{2}=\Delta^{\lambda}\{f+4\}+\Delta^{\lambda+1}\{f+2\}+2 \Delta^{\lambda+2}\{f\}+\Delta^{\lambda+3}\{f-2\}+\Delta^{\lambda+4}\{f-4\} .
$$

The factor 2 in the third term is to indicate that the representation $\Delta^{\lambda+2}\{f\}$ appears twice in this decomposition, at least if $f \geqq 2$; the second, fourth, and fifth terms appear only for $f \geqq 1, f \geqq 3$, and $f \geqq 4$, respectively. The coefficients are the generalized Kronecker symbols, as in the preceding corollary; and since the computations are straightforward and hence uninteresting, further details will be suppressed. It should be noted that for all the second order operators except $\mathscr{D}_{7}$ the coefficients are fully symmetric in the indices corresponding to the differentiations, reflecting the fact that the component $\{4\} \subset \sigma_{2}$ is the term to which the 
operator is associated; the exceptional role played by $\mathscr{D}_{7}$ arises from the fact that that operator is associated to the term $\Delta^{2} \subset \sigma_{2}$. It should also be noted that these explicit formulas for the second order operators can be derived by iterating the first order operators of Corollary 1.1; thus, for instance, $\mathscr{D}_{1} \mathscr{D}_{2}=\mathscr{D}_{4}$.

\section{Holomorphic differential $\mathfrak{G}$-homomorphisms.}

(7) Suppose that $\rho$ and $\hat{\rho}$ are irreducible holomorphic representations of $\mathrm{GL}(n, \mathbb{C})$, and that $\mathscr{D}: \Theta_{\rho} \rightarrow \Theta_{\hat{\rho}}$ is a holomorphic differential $\mathfrak{F}_{\infty}$-homomorphism of degree $v>0$. The operator $\mathscr{D}$ will be written out explicitly as in (1.9). By Theorem 1, all the coefficients are actually constants, and indeed all are zero except those of order $v$; the coefficients of order $v$ will always be supposed to satisfy the symmetry conditions (2.1) and (2.2). If $I=\left(i_{1}, \cdots, i_{v}\right), J=\left(j_{1}, \cdots, j_{v}\right)$, and the abbreviation $A_{i_{1} j_{1} \ldots i_{v} j_{v}}=A_{I J}$ is used, then (1.9) takes the form

$$
(\mathscr{D F})(Z)=\sum_{i \leqq j} A_{I J} \partial^{v} F(Z) / \partial z_{i_{1} j_{1}} \cdots \partial z_{i_{v} j_{v}}
$$

The problem to which the present section is devoted is that of characterizing those $\mathfrak{G}_{\infty}$-homomorphisms, of the above form, which satisfy the more stringent condition of being $\mathbf{5}$-homomorphisms.

THEOREM 2. The holomorphic differential $\mathfrak{G}_{\infty}$-homomorphism $\mathscr{D}: \Theta_{\rho} \rightarrow \Theta_{\hat{\rho}}$ is a (5-homomorphism if and only if

$$
\begin{aligned}
\sum_{i \leqq j} \hat{\rho}(Z) A_{I J} \partial^{v} F(Z) / \partial z_{i_{1} j_{1}} \cdots \partial z_{i_{v} j_{v}} \\
=\sum_{i \leqq j} \sum_{k, l} A_{K L} z_{k_{1} i_{1}} z_{l_{1} j_{1}} \frac{\partial}{\partial z_{i_{1} j_{1}}} \cdots z_{k_{v} i_{v}} z_{l_{v} j_{v}} \frac{\partial}{\partial z_{i_{v} j_{v}}} \rho(Z) F(Z)
\end{aligned}
$$

for all $F(Z) \in \Theta_{\rho}$ and all $Z \in \mathbb{G}$.

Proof. In view of Lemma 1 , and the relevant definitions, it is clear that $\mathscr{D}$ is a (5-homomorphism if and only if

$$
\mathscr{D}\left(\left.F\right|_{\rho} J\right)=\left.(\mathscr{D} F)\right|_{\hat{\rho}} J
$$

for all $F \in \Theta_{\rho}$, where $J \in \mathbb{G}$ is the involution introduced in $\S 1$. Putting $W=J(Z)=-Z^{-1}$, it follows from (1.7) that $\rho(J, Z)=\rho(W)^{-1}$ and that $\hat{\rho}(J, Z)=\hat{\rho}(W)^{-1}$; thus, for instance, $\left(\left.F\right|_{\rho} J\right)(Z)=\rho(W) F(W)$. Note that, furthermore,

$$
\partial w_{i j} / \partial z_{k l}=\sum_{r, s} w_{r i} w_{s j} \partial z_{r s} / \partial z_{k l}=\left(1-\frac{1}{2} \delta_{l}^{k}\right)\left(w_{k i} w_{l j}+w_{k j} w_{l i}\right)
$$

Now on the one hand

$$
\left(\left.(\mathscr{D} F)\right|_{\hat{\rho}} J\right)(Z)=\hat{\rho}(w) \sum_{i \leqq j} A_{I J} \partial^{v} F(W) / \partial w_{i_{1} j_{1}} \cdots \partial w_{i_{v} j_{v}}
$$


and on the other hand

$$
\begin{aligned}
\left(\mathscr{D}\left(\left.F\right|_{\rho} J\right)\right)(Z) & =\sum_{k \leqq l} A_{K L} \partial^{v}(\rho(W) F(W)) / \partial z_{k_{1} l_{1}} \cdots \partial z_{k_{v} l_{v}} \\
& =\sum_{k \leqq l} \sum_{i \leqq j} A_{K L} \frac{\partial w_{i_{1} j_{1}}}{\partial z_{k_{1} l_{1}}} \frac{\partial}{\partial w_{i_{1} j_{1}}} \cdots \frac{\partial w_{i_{v} j_{v}}}{\partial z_{k_{v} l .}} \frac{\partial}{\partial w_{i_{v}, j . .}} \rho(W) F(W) \\
& =\sum_{i \leqq j} \sum_{k, l} A_{K L} w_{k_{1} i_{1}} w_{l_{1} j_{1}} \frac{\partial}{\partial w_{i_{1} j_{1}}} \cdots w_{k_{v} i_{v}} w_{l_{v} j_{v}} \frac{\partial}{\partial w_{i_{v} j_{v}}} \rho(W) F(W) .
\end{aligned}
$$

Thus, after the obvious change of notation, (4.3) is equivalent to (4.2), and the theorem is thereby proved.

If the differentiations indicated in the second term of formula (4.2) are carried out, and the coefficients of the various orders of derivatives of $F(Z)$ are collected and compared, it becomes evident that (4.2) reduces to $v+1$ independent equations, one of which is a trivial identity; hence (4.2) really amounts to $v$ separate conditions. The details of this procedure in the general case are, indeed, onerous. At present the primary interest is in differential operators of first and second degrees; consequently, explicit calculations will be given only for those two cases. To simplify the notation, put $\partial_{i j}=\partial / \partial z_{i j}$. Then note that

$$
\begin{aligned}
\partial_{i j}\left(z_{a c} z_{b d}\right) & =\left(1-\frac{1}{2} \delta_{j}^{i}\right)\left[z_{b d}\left(\delta_{i}^{a} \delta_{j}^{c}+\delta_{j}^{a} \delta_{i}^{c}\right)+z_{a c}\left(\delta_{i}^{b} \delta_{j}^{d}+\delta_{j}^{b} \delta_{i}^{d}\right)\right] \\
& =\left(2-\delta_{j}^{i}\right) \sum_{r, s} z_{a r} z_{b s} \zeta_{r s}{ }^{c d}{ }_{i j}
\end{aligned}
$$

where

$$
2 \zeta_{r s i j}^{c d}=z_{r i}^{-1} \delta_{j}^{c} \delta_{s}^{d}+z_{r j}^{-1} \delta_{i}^{c} \delta_{s}^{d}+z_{s i}^{-1} \delta_{r}^{c} \delta_{j}^{d}+z_{s j}^{-1} \delta_{r}^{c} \delta_{i}^{d}
$$

and hence, $\zeta_{r s}{ }^{c d} i j$ is symmetric in $i$ and $j$.

COROLlaRY 2.1. A holomorphic differential $\mathfrak{G}_{\infty}$-homomorphism $\mathscr{D}$ of order 1 is a (5-homomorphism if and only if:

$$
\sum_{i \leqq j} A_{i j} \rho(Z)^{-1} \partial_{i j} \rho(Z)=0
$$

Proof. In the case of degree $v=1$, equation (4.2) of Theorem 2 has the form

$$
\sum_{i \leqq j} \hat{\rho}(Z) A_{i j} \partial_{i j} F(Z)=\sum_{i \leqq j ; k, i} A_{k l} z_{k i} z_{l j} \partial_{i j} \rho(Z) F(Z)
$$

By using formula (2.10) of Theorem 1, the right-hand side $R$ of equation (4.7) can be written as 


$$
\begin{aligned}
R & =\sum_{i \leqq j} \hat{\rho}(Z) A_{i j} \rho(Z)^{-1} \partial_{i j} \rho(Z) F(Z) \\
& =\hat{\rho}(Z) \sum_{i \leqq j} A_{i j}\left[\rho(Z)^{-1}\left(\partial_{i j} \rho(Z)\right) F(Z)+\partial_{i j} F(Z)\right]
\end{aligned}
$$

Substituting this back into (4.7), it follows that the condition of Theorem 2 takes the form

$$
\hat{\rho}(Z) \sum_{i \leqq j} A_{i j} \rho(Z)^{-1}\left(\partial_{i j} \rho(Z)\right) F(Z)=0 .
$$

Since $\hat{\rho}(Z)$ is nonsingular and $F(Z)$ is arbitrary, (4.6) follows, and the proof is completed.

COROllaRY 2.2. A holomorphic differential $\mathfrak{5}_{\infty}$-homomorphism $\mathscr{D}$ of order 2 is a (5-homomorphism if and only if the following two conditions are both satisfied:

$$
2 \sum_{i_{1} \leqq j_{1} ; i_{2} \leqq j_{2}} A_{i_{1} i_{2} j_{1} j_{2}} z_{i_{1} j_{1}}^{-1} \rho(Z)^{-1} \partial_{i_{2} j_{2}} \rho(Z)
$$

$$
+\sum_{i_{1} \leqq j_{1} ; i_{2} \leqq j_{2}} A_{i_{1} j_{1} i_{2} j_{2}} \rho(Z)^{-1} \partial_{i_{1} j_{1}} \partial_{i_{2} j_{2}} \rho(Z)=0
$$

and

$$
\sum_{i_{1} j_{1}} A_{i_{1} i_{2} j_{1} j_{2}} z_{i_{1} j_{1}}^{-1}+\sum_{i_{1} \leqq j_{1}} A_{i_{1} j_{1} i_{2} j_{2}} \rho(Z)^{-1} \partial_{i_{1} j_{1}} \rho(Z)=0
$$

all $i_{2}, j_{2}$.

Proof. In the case $v=2$, equation (4.2) of Theorem 2 has the form

$$
\begin{aligned}
& \sum_{i \leqq j} \hat{\rho}(Z) A_{I J} \partial_{i_{1} j_{1}} \partial_{i_{2} j_{2}} F(Z) \\
&=\sum_{i \leqq j ; k, l} A_{K L} z_{k_{1} i_{1}} z_{l_{1} j_{1}} \partial_{i_{1} j_{1}} z_{k_{2} i_{2} i_{2}} z_{l_{2} j_{2}} \partial_{i_{2} j_{2}} \rho(Z) F(Z) .
\end{aligned}
$$

Recalling (4.4), the right-hand side $R$ of (4.10) can be rewritten:

$$
\begin{aligned}
R= & \sum_{i \leqq j ; k, l, r, s}\left(2-\delta_{j_{1}}^{i_{1}}\right) A_{K L} z_{k_{1} i_{1}} z_{l_{1} j_{1}} z_{k_{2} r} z_{l_{2} s} \zeta_{r s}{ }^{i_{2} j_{2}}{ }_{i_{1} j_{1}} \partial_{i_{2} j_{2}} \rho(Z) F(Z) \\
& +\sum_{i \leqq j ; k, l} A_{K L} z_{k_{1} i_{1}} z_{l_{1} j_{1}} z_{k_{2} i_{2}} z_{l_{2} j_{2}} \partial_{i_{1} j_{1}} \partial_{i_{2} j_{2}} \rho(Z) F(Z) \\
= & R_{1}+R_{2} .
\end{aligned}
$$

Applying condition (2.10) of Theorem 1, and rewriting in the obvious manner, secure that 


$$
R_{1}=\sum_{i_{2} \leqq j_{2}} \sum_{i_{1} j_{1} r s} \hat{\rho}(Z) A_{i_{1} j_{1} r s} \zeta_{r s}{ }_{i_{2} j_{2}}^{i_{1} j_{1}} \rho(Z)^{-1} \partial_{i_{2} j_{2}} \rho(Z) F(Z)
$$

but by (4.5) this reduces to

$$
R_{1}=2 \sum_{i_{1} j_{1} ; i_{2} \leqq j_{2}} \hat{\rho}(Z) A_{i_{1} i_{2} j_{1} j_{2}} z_{i_{1} j_{1}}^{-1} \rho(Z)^{-1} \partial_{i_{2} j_{2}} \rho(Z) F(Z) .
$$

By another application of (2.10), observe that

$$
R_{2}=\sum_{i \leqq j} \hat{\rho}(Z) A_{i_{1} j_{1} i_{2} j_{2}} \rho(Z)^{-1} \partial_{i_{1} j_{1}} \partial_{i_{2} j_{2}} \rho(Z) F(Z) .
$$

Substituting these expressions for $R_{1}$ and $R_{2}$ back into $R$, performing the indicated differentiations, and collecting terms together, secure that

$$
\begin{aligned}
& R= \hat{\rho}(Z)\left\{2 \sum_{i_{1} j_{1} ; i_{1} \leqq j_{2}} A_{i_{1} i_{2} j_{1} j_{2}} z_{i_{1} j_{1}}^{-1} \rho(Z)^{-1}\left(\partial_{i_{2} j_{2}} \rho(Z)\right)\right. \\
&\left.+\sum_{i \leqq j} A_{i_{1} j_{1} i_{2} j_{2}} \rho(Z)^{-1}\left(\partial_{i_{1} j_{1}} \partial_{i_{2} j_{2}} \rho(Z)\right)\right\} F(Z) \\
&+2 \hat{\rho}(Z) \sum_{i_{2} \leqq j_{2}}\left\{\sum_{i_{1} j_{1}} A_{i_{1} i_{2} j_{1} j_{2}} z_{i_{1} j_{1}}^{-1}\right. \\
&\left.+\sum_{i_{1} \leqq j_{1}} A_{i_{1} j_{1} i_{2} j_{2}} \rho(Z)^{-1}\left(\partial_{i_{1} j_{1}} \rho(Z)\right)\right\} \partial_{i_{2} j_{2}} F(Z) \\
&+\hat{\rho}(Z) \sum_{i \leqq j} A_{i_{1} j_{1} i_{2} j_{2}} \partial_{i_{1} j_{1}} \partial_{i_{2} j_{2}} F(Z) .
\end{aligned}
$$

Upon substituting the above expression for $R$ back into (4.10), and recalling that $\hat{\rho}(Z)$ is nonsingular and that $F(Z)$ is an arbitrary function, conditions (4.6) follow immediately, and the proof is thereby completed.

For some purposes it is useful to rewrite the criterion of Corollary $2.2 \mathrm{in}$ a form which involves only the first derivatives of the representation function $\rho(Z)$, and, indeed, in which these derivatives appear as logarithmic derivations.

COROllaRy 2.3. A holomorphic differential $\mathfrak{5}_{\infty}$-homomorphism $\mathscr{D}$ of order 2 is a (5-homomorphism if and only if condition (4.9) and the following are both satisfied:

$$
\frac{1}{2} \sum_{i_{1} j_{1} i_{2} j_{2}}\left(A_{i_{1} j_{1} i_{2} j_{2}}+A_{i_{1} i_{2} j_{1} j_{2}}\right) z_{i_{1} j_{1}}^{-1} z_{i_{2} j_{2}}^{-1}
$$

$$
+\sum_{i_{1} j_{1} ; i_{2} \leqq j_{2}} A_{i_{1} i_{2} j_{1} j_{2}} z_{i_{1} j_{1}}^{-1}\left(\rho(Z)^{-1} \partial_{i_{2} j_{2}} \rho(Z)\right)=0 .
$$

Proof. It is of course sufficient to show that (4.8) and (4.9) are together equivalent to (4.9) and (4.11). Upon differentiating (4.9) with respect to $z_{i_{2} j_{2}}$, and summing over the indices $i_{2} \leqq j_{2}$, it follows that 


$$
\begin{aligned}
\sum_{i_{1} \leqq j_{1} ; i_{2} \leqq j_{2}} A_{i_{1} j_{1} i_{2} j_{2}} \rho(Z)^{-1} \partial_{i_{1} j_{1}} \partial_{i_{2} j_{2}} \rho(Z) & \\
= & \sum_{i_{1} \leqq j_{1} ; i_{2} \leqq j_{2}} A_{i_{1} j_{1} i_{2} j_{2}}\left(\rho(Z)^{-1} \partial_{i_{1} j_{1}} \rho(Z)\right)\left(\rho(Z)^{-1} \partial_{i_{2} j_{2}} \rho(Z)\right) \\
& +\frac{1}{2} \sum_{i_{1} j_{1} i_{2} j_{2}}\left(A_{i_{1} j_{1} i_{2} j_{2}}+A_{i_{1} i_{2} j_{1} j_{2}}\right) z_{i_{1} j_{1}}^{-1} z_{i_{2} j_{2}}^{-1}
\end{aligned}
$$

Again applying (4.9), the above expression becomes

$$
\begin{aligned}
= & -\sum_{i_{1} j_{1}} \sum_{i_{2} \leqq j_{2}} A_{i_{1} i_{2} j_{1} j_{2}} z_{i_{1} j_{1}}^{-1} \rho(Z)^{-1} \partial_{i_{2} j_{2}} \rho(Z) \\
& +\frac{1}{2} \sum_{i_{1} j_{1} i_{2} j_{2}}\left(A_{i_{1} j_{1} i_{2} j_{2}}+A_{i_{1} i_{2} j_{1} j_{2}}\right) z_{i_{1} j_{1}}^{-1} z_{i_{2} j_{2}}^{-1}
\end{aligned}
$$

and since substituting this into (4.8) yields (4.11), the proof is thereby completed.

REMARK. If the coefficients $A_{i_{1} j_{1} i_{2} j_{2}}$ are symmetric in the indices $i_{1}, j_{1}, i_{2}, j_{2}$, then (4.11) in turn follows from (4.9), so that the latter condition suffices by itself.

The calculations for differential operators of any degree $v$ follow the same general pattern but with increasing technical complications. To conclude this section, the results for differential operators of degree 3 will be listed, with details of the proof suppressed.

COROLlaRY 2.4. A holomorphic differential $\mathfrak{G}_{\infty}$-homomorphism $\mathscr{D}$ of order 3 is a (5-homomorphism if and only if the following three conditions are all satisfied:

$6 \sum_{i_{1} j_{1} i_{2} j_{2}} \sum_{i_{3} \leqq j_{3}} A_{i_{1} i_{2} i_{3} j_{1} j_{2} j_{3}} z_{i_{1} j_{1}}^{-1} z_{i_{2} j_{2}}^{-1} \rho(Z)^{-1} \partial_{i_{3} j_{3}} \rho(Z)$

$$
\begin{aligned}
& +6 \sum_{i_{1} j_{1}} \sum_{i_{2} \leqq j_{2} ; i_{3} \leqq j_{3}} A_{i_{1} i_{2} j_{1} j_{2} i_{3} j_{3}} z_{i_{1} j_{1}}^{-1} \rho(Z)^{-1} \partial_{i_{2} j_{2}} \partial_{i_{3} j_{3}} \rho(Z) \\
& +\sum_{i \leqq j} A_{i_{1} j_{1} i_{2} j_{2} i_{3} j_{3}} \rho(Z)^{-1} \partial_{i_{1} j_{1}} \partial_{i_{2} j_{2}} \partial_{i_{3} j_{3}} \rho(Z)=0
\end{aligned}
$$

$2 \sum_{i_{1} j_{1} i_{2} j_{2}} A_{i_{1} i_{2} i_{3} j_{1} j_{2} j_{3}} z_{i_{1} j_{1}}^{-1} z_{i_{2} j_{2}}^{-1}$

$$
\begin{aligned}
& +2 \sum_{i_{1} j_{1}} \sum_{i_{2} \leqq j_{2}}\left(A_{i_{1} i_{2} j_{1} j_{2} i_{3} j_{3}}+A_{i_{1} i_{3} j_{1} j_{3} i_{2} j_{2}}\right) z_{i_{1} j_{1}}^{-1} \rho(Z)^{-1} \partial_{i_{2} j_{2}} \rho(Z) \\
& +\sum_{i \leqq j} A_{i_{1} j_{1} i_{2} j_{2} i_{3} j_{3}} \rho(Z)^{-1} \partial_{i_{1} j_{1}} \partial_{i_{2} j_{2}} \rho(Z)=0 \quad \text { for all } i_{3}, j_{3}
\end{aligned}
$$

$$
2 \sum_{i_{1} j_{1}} A_{i_{1} i_{2} j_{1} j_{2} i_{3} j_{3}} z_{i_{1} j_{1}}^{-1}+\sum_{i_{1} \leqq j_{1}} A_{i_{1} j_{1} i_{2} j_{2} i_{3} j_{3}}^{\prime} \rho(Z)^{-1} \partial_{i_{1} j_{1}} \rho(Z)=0 \text { for all } i_{2} j_{2} i_{3} j_{3} .
$$


(8) By the use of a more explicit form for the representation $\rho$, the preceding corollaries can be rewritten in a simpler manner, so as to express the criterion for

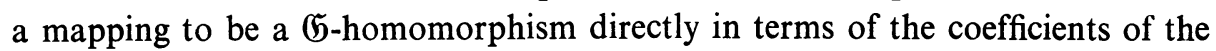
differential operator. Suppose that the representation $\rho$ is given by the symmetry scheme $\rho=\Delta^{\lambda}\left\{f_{1}, \cdots, f_{r}\right\}$, and that $f=f_{1}+\cdots+f_{r}$; the representation space $V_{\rho}$ hence consists of tensors $\xi_{j_{1} \ldots j_{f}}$ of total degree $f$ which have the appropriate symmetries in the indices $j_{1}, \cdots, j_{f}$, as in [12] or [1]. Suppose further that $A_{r s}$ is a set of linear mappings from $V_{\rho}$ to some other vector space, for $1 \leqq r, s \leqq n$. If $\xi=\left\{\xi_{j_{1} \ldots j_{f}}\right\} \in V_{\rho}$, then in terms of these components the mappings $A_{r s}$ can be written: $A_{r s} \cdot \xi=\sum_{J} A_{j_{1} \ldots j_{j} r s} \xi_{j_{1} \ldots j_{f}}$, where each $A_{j_{1} \ldots j_{f} r s}$ is a vector in the image of $A_{r s}$.

LEMMA 6. If $A_{r s}=A_{s r}$, then for any $\xi \in V_{\rho}$,

$$
\begin{aligned}
& \left(\sum_{r \leqq s} A_{r s} \rho(Z)^{-1} \partial_{r s} \rho(Z)\right) \cdot \xi \\
& \quad=\sum_{K} \sum_{r, s}\left(\lambda A_{k_{1} \ldots k_{f} r s}+\sum_{e=1}^{f} A_{k_{1} \ldots s \ldots k_{g} r k_{e}}\right) z_{r s}^{-1} \xi_{k_{1} \ldots k_{f}} .
\end{aligned}
$$

Proof. Recall that $\partial_{r s} \operatorname{det} Z=\left(2-\delta_{s}^{r}\right) z_{r s}^{-1} \operatorname{det} Z$. Then, by a straightforward calculation,

$$
\begin{aligned}
& \sum_{r \leqq s} A_{r s} \rho(Z)^{-1} \partial_{r s} \rho(Z) \cdot \xi \\
& \quad=\sum_{I J K} \sum_{r \leqq s} A_{i_{1} \ldots i_{f} r s} \operatorname{det} Z^{-\lambda} z_{i_{1} j_{1}}^{-1} \cdots z_{i_{f} j_{f}}^{-1} \cdot \partial_{r s}\left(\operatorname{det} Z^{\lambda} z_{j_{1} k_{1}} \cdots z_{j_{f} k_{f}}\right) \cdot \xi_{k_{1} \ldots k_{f}} \\
& \quad=\sum_{I J K} \sum_{r \leqq s} A_{i_{1} \ldots i_{f} r s} \operatorname{det} Z^{-\lambda} z_{i_{1} j_{1}}^{-1} \cdots z_{i_{f} j_{f}}^{-1} \cdot\left\{\lambda\left(2-\delta_{s}^{r}\right) z_{r s}^{-1} \operatorname{det} Z^{\lambda} z_{j_{1} k_{1}} \cdots z_{j_{f} k}\right. \\
& \left.\quad+\sum_{e=1}^{s} \frac{1}{2}\left(2-\delta_{s}^{r}\right)\left(\delta_{j_{e}}^{r} \delta_{k_{e}}^{s}+\delta_{k_{e}}^{r} \delta_{j_{e}}^{s}\right) \operatorname{det} Z^{\lambda} z_{j_{1} k_{1}} \cdots \hat{z}_{j_{e} k_{e}} \cdots z_{j_{f} k_{f}}\right\} \cdot \xi_{k_{1} \ldots k_{f}},
\end{aligned}
$$

(where $\hat{z}_{j k}$ means that that term is to be omitted),

$$
=! \sum_{K} \sum_{r, s}\left\{A_{k_{1} \ldots k_{f} r s} \lambda z_{r s}^{-1}+\sum_{e=1}^{s} \sum_{i_{e} j_{e}} A_{k_{1} \ldots i_{e} \ldots k_{f} r s} z_{i_{e} j_{e}}^{-1} \frac{1}{2}\left(\delta_{j_{e}}^{r} \delta_{k_{e}}^{s}+\delta_{k_{e}}^{r} \delta_{j_{e}}^{s}\right)\right\} \xi_{k_{1} \ldots k_{f}},
$$

which reduces immediately to the right-hand side of (4.15), and thus concludes the proof.

Now the coefficients $A_{I J}$ in the differential operator $\mathscr{D}$ of (4.1) can be considered as linear maps from $V_{\rho}$ to $V_{\hat{\rho}}$, and can therefore be written as above; an application of Lemma 6 then leads to the following 
COROLlaRY 2.5. A holomorphic differential $\mathfrak{G}_{\infty}$-homomorphism $\mathscr{D}$ of order 1 is a (5-homomorphism if and only if

$$
\lambda A_{k_{1} \ldots k_{f} i j}+\sum_{e=1}^{f} A_{k_{1} \ldots j \ldots k_{j} i k_{e}}
$$

is skew-symmetric in $(i, j)$.

Proof. Upon substituting (4.15) into (4.6), the criterion of Corollary 2.1 reduces to the assertion that the expression (4.16), when multiplied by $z_{i j}^{-1}$ and summed over all values $1 \leqq i, j \leqq n$, yields zero; since this must hold for an arbitrary symmetric matrix $z_{i j}^{-1}$, it follows that (4.16) must be skew-symmetric, as asserted.

COROLLARY 2.6. A holomorphic differential $\mathfrak{b}_{\infty}$-homomorphism $\mathscr{D}$ of order 2 is a (5-homomorphism if and only if the following two conditions are both satisfied:

$$
A_{k_{1} \ldots k_{f} i_{1} i_{2} j_{1} j_{2}}+\lambda A_{k_{1} \ldots k_{f} i_{1} j_{1} i_{2} j_{2}}+\sum_{e=1}^{f} A_{k_{1} \ldots j_{1} \ldots k_{f} i_{1} k_{e} i_{2} j_{2}}
$$

is skew-symmetric in $\left(i_{1}, j_{1}\right)$ for each fixed $\left(i_{2}, j_{2}\right)$; and

$$
\begin{aligned}
\frac{1}{2}\left(A_{k_{1} \ldots k_{f} i_{1} j_{1} i_{2} j_{2}}+A_{k_{1} \ldots k_{f} i_{1} i_{2} j_{1} j_{2}}\right) & +\lambda A_{k_{1} \ldots k_{f} i_{1} i_{2} j_{1} j_{2}} \\
& +\sum_{e=1}^{f} A_{k_{1} \ldots j_{2} \ldots k_{f} i_{1} i_{2} j_{1} k_{e}}
\end{aligned}
$$

is sufficiently skew-symmetric to annihilate the representation $\sigma_{2}$.

ReMARK. For each fixed set of indices $k_{1} \cdots k_{f}$ the expression (4.18) can be considered as a linear transformation from the representation space $V_{2}$ of the representation $\sigma_{2}$ to $V_{\hat{\rho}}$, where $V_{2}$ consists of the tensors $\xi_{i_{1} j_{1} i_{2} j_{2}}$ with the symmetry properties (2.1) and (2.2); the second condition above is that this transformation be the zero mapping. As noted earlier, if $A_{k_{1} \ldots k_{f} i_{1} j_{1} i_{2} j_{2}}$ is fully symmetric in $i_{1} j_{1} i_{2} j_{2}$ then the first condition of this corollary automatically implies the second condition.

Proof. Once again a direct application of Lemma 6 shows that (4.9) is equivalent to the vanishing of the product of (4.17) with $z_{i_{1} j_{1}}^{-1}$, summed over all $1 \leqq i_{1}$, $j_{1} \leqq n$; and that (4.11) is equivalent to the vanishing of the product of (4.18) with $z_{i_{1} j_{1}}^{-1} z_{i_{2} j_{2}}^{-1}$, summed over all $1 \leqq i_{1}, j_{1}, i_{2}, j_{2} \leqq n$. Thus the conclusion of Corollary 2.6 follows directly from that of Corollary 2.3 itself.

\section{Some examples of $\mathfrak{5}$-homomorphisms.}

(9) Returning once again to the case $n=2$, the results obtained in the preceding section will be applied to determine which of the $\mathfrak{G}_{\infty}$-homomorphisms 
of $\$ 3$ are 5 -homomorphisms as well. For first order differential operators the conclusions follow quite directly from Corollary 2.5; since, however, these operators were discussed in [6], only the results will be listed here for reference. Thus, the operators $\mathscr{D}_{1}, \mathscr{D}_{2}, \mathscr{D}_{3}$ of Corollary 1.1 are $(5$-homomorphisms if and only if the following conditions are fulfilled, respectively:

$$
\left\{\begin{array}{l}
\text { for } \mathscr{D}_{1}: \lambda=-f ; \\
\text { for } \mathscr{D}_{2}: \lambda=1-\frac{1}{2} f ; \\
\text { for } \mathscr{D}_{3}: \lambda=1
\end{array}\right.
$$

For the remainder of this section, then, only the second degree operators will be considered; the conclusions are as follows:

THEOREM 3. For the symplectic group $\mathfrak{G}$ on the Siegel generalized upper half-plane of rank 2, and the irreducible holomorphic representations $\rho=\Delta^{\lambda}\{f\}$, $\hat{\rho}=\Delta^{\hat{\lambda}}\{\hat{f}\}$ of $\mathrm{GL}(2, \mathbb{C})$, there are holomorphic differential (5-homomorphisms $\mathscr{D}: \Theta_{\rho} \rightarrow \Theta_{\hat{\rho}}$ of second order of the following forms, and no others:

(5.2) for $f \geqq 0, \lambda=-(f+1)$, and $\hat{\rho}=\Delta^{\lambda}\{f+4\}$, the operator $\mathscr{D}_{4}$ of (3.7);

(5.3) for $f \geqq 4, \lambda=0$, and $\hat{\rho}=\Delta^{4}\{f-4\}$, the operator $\mathscr{D}_{9}$ of (3.11).

Proof. It is of course only necessary to consider those differential operators which are already $\mathfrak{G}_{\infty}$-homomorphisms, as determined in Corollary 1.2. Using the explicit forms for the representations $\rho$ and $\hat{\rho}$, as in $\S 4$, the differential operators can all be written as follows:

$$
(\mathscr{D} F)_{i_{1} \ldots \rho i+4}
$$

$$
=\sum_{j_{1} \ldots j_{f}} \sum_{j_{f+1} \leqq j_{f+2} ; j_{f+3} \leqq j_{f+4}} A_{i_{1} \ldots i_{f+4} ; j_{1} \ldots j_{f+4}} \partial^{2} F_{j_{1} \ldots j_{f}} / \partial z_{j_{f+1} j_{f+2}} \partial z_{j_{f+3} j_{f+4}} .
$$

The additional requirement that the operator $\mathscr{D}$ be a $\mathbf{5}$-homomorphism is then as in Corollary 2.6; and it is merely necessary to determine which of the operators of Corollary 1.2 satisfy conditions (4.17) and (4.18).

To begin, consider the representations $\hat{\rho}=\Delta^{\lambda}\{f+4\}, \hat{\rho}=\Delta^{\lambda+1}\{f+2\}$, $\hat{\rho}=\Delta^{\lambda+3}\{f-2\}$, and $\hat{\rho}=\Delta^{\lambda+4}\{f-4\}$. In each of these cases there is a unique holomorphic $\mathfrak{G}_{\infty}$-homomorphism $\mathscr{D}: \Theta_{\rho} \rightarrow \Theta_{\hat{\rho}}$, and the coefficients in (5.4) are, except for scalar multiples and change of basis, the generalized Kronecker symbols

$$
A_{I ; J}=\delta\left[I\left|j_{1} \cdots j_{f}\right| j_{f+1} j_{f+2} j_{f+3} j_{f+4}\right]=\delta_{J}^{I}
$$

introduced in [6]; the indices $I$ in (5.5) are to be arranged in the appropriate 
symmetry scheme, depending on the particular representation $\hat{\rho}$. Since these are all fully symmetric in the last four indices $j_{f+1}, \cdots, j_{f+4}$, only condition (4.17) need be considered. That condition can be expressed more conveniently by introducing some additional permutations on the indices $j_{1}, \cdots, j_{f+4}$; thus, for $1 \leqq e \leqq f+4$, let $\lambda_{e}$ be the permutation which interchanges the indices $j_{e}$ and $j_{f+1}$, and to simplify writing, put $\lambda_{f+2}=\alpha, \lambda_{f+3}=\beta, \lambda_{f+4}=\gamma$. In addition to the Kronecker symbol $\delta_{J}^{I}$ of (5.5), let

$$
\zeta_{J}^{I}=\sum_{e=1}^{f} \delta_{\lambda_{e} J}^{I} \quad \text { and } \quad \xi_{J}^{I}=\zeta_{J}+\zeta_{\alpha J}^{I} .
$$

Condition (4.17) then reduces to the assertion that $(1+\lambda) \delta_{J}{ }^{I}+\zeta_{J}^{I}$ be skewsymmetric in $j_{f+1}, j_{f+2}$; or what is the same thing, that

$$
2(1+\lambda) \delta_{J}^{I}+\xi_{J}^{I}=0
$$

To investigate this, introduce the further tensors $+\zeta_{J}^{I}=\zeta_{J}^{I}+\zeta_{\alpha J}^{I}+\zeta_{\beta J}^{I}+\zeta_{\gamma J}^{I}$; ${ }^{1} \zeta_{J}^{I}=\zeta_{J}^{I}-\zeta_{\alpha J}^{I} ;{ }^{2} \zeta_{J}^{I}=\zeta_{J}^{I}-\zeta_{B J}^{I} ;$ and ${ }^{3} \zeta_{J}^{I}=\zeta_{J}^{I}-\zeta_{\gamma J}^{I}$. Recall that the tensor $\zeta_{J}^{I}$ has the symmetries of $\{f\} \otimes\{1\} \otimes\{3\}$ in the indices $J$; hence the tensor $+\zeta_{J}^{I}$ has the symmetry of $\{f\} \otimes\{4\}$, while the tensors ${ }^{k} \zeta_{J}^{I}(k=1,2,3)$ have the symmetries of $\{f\} \otimes\{3,1\}$, after suitable permutations. It then follows immediately from Schur's lemma, as in the argument in Theorem 1 of [6], that these tensors are in turn scalar multiples of the generalized Kronecker tensors of the appropriate symmetries. Thus, for instance

$$
+\zeta_{J}^{I}=k \cdot \delta_{J}^{I} \text { and }{ }^{1} \zeta_{J}^{I}=k_{1} \cdot \tilde{\delta}_{\pi_{1} J}^{I}=k_{1} \cdot \delta\left[I\left|j_{1} \cdots j_{f}\right|_{j_{f}+1}^{j_{f}+{ }_{2} j_{f}+{ }_{3} j_{f}++_{4}}\right] .
$$

On the other hand, it follows immediately from the preceding definitions that $\xi_{J}^{I}=+\zeta_{J}^{I}-\zeta_{\beta J}^{I}-\zeta_{\gamma J}^{I}=\frac{1}{2}+\zeta_{J}^{I}-\frac{1}{2} 1 \zeta_{J}^{I}+\frac{1}{2} 2 \zeta_{J}^{I}+\frac{1}{2}{ }^{3} \zeta_{J}^{I}$; consequently, the tensor $\xi_{J}^{I}$ can be decomposed into the primitive Kronecker symbols

$$
\begin{aligned}
& \xi_{J}^{I}=c \delta_{J}^{I}+c_{1} \tilde{\delta}_{\pi_{1} J}^{I}+c_{2} \tilde{\delta}_{\pi_{2} J}^{I}+c_{3} \tilde{\delta}_{\pi_{3} J}^{I}
\end{aligned}
$$

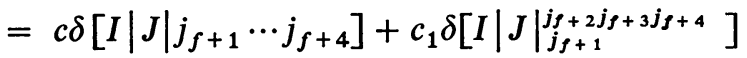

$$
\begin{aligned}
& +c_{2} \delta\left[I|J|_{j_{f}+1}^{j_{f}+{ }_{3} j_{f}+{ }_{2} j_{f}+4}\right]+c_{3} \delta\left[I|J|_{j_{f+1}}^{j_{f+4} j_{f}+{ }_{2} j_{f+3}}\right],
\end{aligned}
$$

for some constants $c, c_{1}, c_{2}, c_{3}$ depending on $\lambda$ and on $\{f\}$. Thus it follows directly that condition (5.7) reduces to the assertion that $2(1+\lambda)+c=0, c_{1}=c_{2}=c_{3}=0$; and it merely remains to compute the numerical values of the coefficients c. $c_{1}, c_{2}, c_{3}$. This can, of course, be done by substituting particular sets of indices $I, J$ into (5.9), computing the values of the tensors by recalling the definition of the generalized Kronecker tensors (equation (1.20) in [6]), and solving the 
resulting set of linear equations in $c, c_{1}, c_{2}, c_{3}$. Since this is a straightforward calculation, the details will be omitted, except for the following observation. For the representations $\hat{\rho}=\Delta^{\lambda}\{f+4\}$ and $\hat{\rho}=\Delta^{\lambda+4}\{f-4\}$ it is evident, from Lemma 4, that $\hat{\rho} \notin \rho \otimes\{3,1\}=\rho \otimes \Delta\{2\}$; hence in these cases $\tilde{\delta}_{J}^{I}=0$, so that $c_{1}=c_{2}=c_{3}=0$ without further ado. The results of the calculation can be summarized as follows:

$$
\begin{aligned}
& \text { if } \hat{\rho}=\Delta^{\lambda}\{f+4\} \quad \text { then } \quad c=2 f, \quad c_{1}=c_{2}=c_{3}=0 \text {; } \\
& \text { if } \hat{\rho}=\Delta^{\lambda+1}\{f+2\} \text { then } c=\frac{1}{2}(3 f-4), c_{1}=\frac{1}{2} f \text {, } \\
& c_{2}=c_{3}=-\frac{1}{2} f
\end{aligned}
$$

$$
\text { if } \hat{\rho}=\Delta^{\lambda+3}\{f-2\} \text { then } \quad \begin{aligned}
c & =\frac{1}{2}(f-6), \quad c_{1}=\frac{3}{2}(f-2), \\
c_{2} & =c_{3}=-\frac{3}{2}(f-2) ;
\end{aligned}
$$

if $\hat{\rho}=\Delta^{\lambda+4}\{f-4\}$ then $c=-2, \quad c_{1}=c_{2}=c_{3}=0$.

Thus the first and last lines of (5.10) give the operators (5.2) and (5.3) of Theorem 3; and, since $f \geqq 1$ for $\hat{\rho}=\Delta^{\lambda+1}\{f+2\}$ and $f \geqq 3$ for $\hat{\rho}=\Delta^{\lambda+3}\{f-2\}$, the remaining two lines of (5.10) lead to no $\mathfrak{6}$-homomorphisms.

Next consider the representation $\hat{\rho}=\Delta^{\lambda+2}\{f\}$. There are two linearly independent $\mathfrak{G}_{\infty}$-homomorphisms $\mathscr{D}: \Theta_{\rho} \rightarrow \Theta_{\hat{\rho}}$, corresponding to the two components $\{4\}$ and $\{2,2\}=\Delta^{2}$ of $\sigma_{2}$; thus the coefficients of the general $\mathfrak{F}_{\infty}$-homomorphism have the form

$$
\begin{aligned}
& A_{I ; J}=a \delta\left[\left[\begin{array}{l}
i_{1} i_{2} \ldots i_{f+2} \\
i_{f+3} i_{f+4}
\end{array}\left|j_{1} \cdots j_{f}\right| j_{f+1} \cdots j_{f+4}\right]\right. \\
& +b \delta\left[\begin{array}{c}
i_{1} i_{2} \ldots i_{f+2} \\
i_{f+3} i_{f+4}
\end{array}\left|j_{1} \cdots j_{f}\right| \begin{array}{l}
j_{f+1} j_{f+2} \\
j_{f+3} j_{f+4}
\end{array}\right] \\
& +b \delta\left[\begin{array}{c}
i_{1} i_{2} \ldots i_{f+2} \\
i_{f+3} i_{f+4}
\end{array}\left|j_{1} \cdots j_{f}\right| \begin{array}{c}
j_{f+1} j_{f+2} \\
j_{f+4} j_{f+3}
\end{array}\right],
\end{aligned}
$$

for arbitrary complex constants $a, b$. The first condition that the mapping be a (5-homomorphism, condition (4.17) in Corollary 2.6, has the form:

$$
2 \lambda A_{I ; J}+A_{I ; \beta J}+A_{I ; \gamma \mathrm{J}}+\sum_{e=1}^{f}\left(A_{I ; \lambda_{e} \mathrm{~J}}+A_{I ; \lambda_{e} \alpha \mathrm{J}}\right)=0,
$$

where $\alpha, \beta, \gamma, \lambda_{e}$ are the permutations introduced above. It is evident that this condition applies independently to the two components in (5.11). For the first component, the argument used in the preceding paragraph of this proof applies, and a straighforward calculation shows that necessarily $a=0$. For the second component, the above condition reduces to the form 


$$
0=(2 \lambda-1)\left\{\delta\left[\left.I|J|\right|_{j_{f}+3 j_{f}+4} ^{j_{f}+{ }_{1} j_{f}+2}\right]+\delta\left[I|J|_{j_{f}+4_{j}+3}^{j_{f}+j_{f}+2}\right]\right\}+\zeta_{I ; J}+\zeta_{I ; \alpha J}
$$

where

$$
\zeta_{I ; J}=\sum_{e=1}^{f}\left\{\delta\left[I\left|j_{1} \cdots j_{f+1} \cdots j_{f}\right|_{j_{f}+3 j_{f}+4}^{j_{e} j_{f}+2}\right]+\delta\left[I\left|j_{1} \cdots j_{f+1} \cdots j_{f}\right|_{j_{f}+j_{j} j_{f}+3}^{j_{e} j_{f}+2}\right]\right\} .
$$

Upon substituting some explicit values for the indices $I, J$, a straightforward calculation shows that necessarily $\lambda=\frac{1}{2}$ and $f=0$, if $b \neq 0$. There are no admissible representations in this case, since $\lambda$ is restricted to assuming only integer values.

REMARK. The suggestion implicit in the last case considered in the above proof, that it may be possible to introduce nonintegral values of $\lambda$ and thereby to secure an additional admissible differential operator, can be pursued as follows. Since the space $\mathfrak{S}$ is simply-connected, then for any integer $r$ and any fixed element $T \in(5)$ there are two well-defined single-valued branches of the function $\operatorname{det}(C Z+D)^{r+1 / 2}$. It is possible to verify, by a simple direct calculation using the methods introduced above, that for suitable choices of these branches it is true that

$$
\mathscr{D}_{7}\left[\operatorname{det}(C Z+D)^{-1 / 2} F(T Z)\right]=\operatorname{det}(C Z+D)^{-5 / 2}\left(\mathscr{D}_{7} F\right)(T Z) .
$$

Hence for any discrete subgroup $\Gamma \subset\left(\mathfrak{H}\right.$ there are branches of $\Delta(T, Z)^{1 / 2}$ and of $\Delta(T, Z)^{5 / 2}$ for which there is a $\Gamma$-homomorphism of the form

$$
\mathscr{D}_{7}: \Theta_{\Delta^{1 / 2}} \rightarrow \Theta_{\Delta^{5 / 2}}
$$

(10) There are three of the second order differential (5-homomorphisms of Theorem 3 which are associated in some manner with one-dimensional representations, that is to say, with ordinary scalar functions; a slightly more detailed discussion of these will perhaps illustrate some of the applications possible in the general case.

The first of these is the operator $\mathscr{D}_{4}$, as in (5.2), for the value $f=0$; this furnishes a (5-homomorphism

$$
\mathscr{D}_{4}: \Theta_{\Delta^{-1}} \rightarrow \Theta_{\Delta^{-1}\{4\}} \text {. }
$$

Both the kernel and the image of $\mathscr{D}_{4}$ are subspaces which are preserved under the appropriate actions by the full symplectic group or any of its subgroups; and the restrictions to these subspaces lead to additional representations of these groups. The image, which is a space of vector-valued functions, can, by considering the appropriate integrability conditions, be characterized as the set of solutions of a further family of differential operators; but these operators are not $\mathfrak{6}$-homomorphisms as considered here, so this topic will not be pursued any further just at present. The kernel is the subspace of scalar functions satisfying $\mathscr{D}_{4}(F)=0$; 
referring to (3.7), the functions in the kernel are just the functions which satisfy the following systems of partial differential equations:

$$
\begin{aligned}
0=\partial^{2} f / \partial z_{11}^{2} & =\partial^{2} f / \partial z_{11} \partial z_{12}=\partial^{2} f / \partial z_{12} \partial z_{22} \\
& =\partial^{2} f / \partial z_{22}^{2}=2 \partial^{2} f / \partial z_{11} \partial z_{22}+\partial^{2} f / \partial z_{12}^{2}
\end{aligned}
$$

A trivial calculation shows that such a function must have the form:

$$
f(z)=c \operatorname{det} Z+c_{11} z_{11}+c_{12} z_{12}+c_{22} z_{22}+c_{0},
$$

for arbitrary complex constants $c$. It is, of course, obvious that the set of functions of the form (5.14) is indeed preserved under the appropriate action of the symplectic group (5. Letting $\mathscr{K} \subset \Theta_{\Delta^{-1}}$ be the kernel of $\mathscr{D}_{4}$, the set of functions of the form (5.14), and $\Xi \subset \Theta_{\Delta^{-1}\{4\}}$ be the image of $\mathscr{D}_{4}$, there corresponds to the exact sequence of $\left(\mathfrak{5}\right.$-spaces $0 \rightarrow \mathscr{K} \rightarrow \Theta_{\Delta^{-1}} \rightarrow^{\mathscr{D}_{4}} \Xi \rightarrow 0$ an exact cohomology sequence generalizing that introduced by Eichler (see [5]), beginning:

$$
0 \rightarrow H^{0}(\Gamma, \mathscr{K}) \rightarrow H^{0}\left(\Gamma, \Theta_{\Delta^{-1}}\right) \rightarrow H^{0}(\Gamma, \Xi) \rightarrow H^{1}(\Gamma, \mathscr{K}) \rightarrow \cdots,
$$

where $\Gamma$ is any subgroup of the symplectic group $(\mathfrak{b}$. In particular, for the symplectic modular group $\Gamma$, as defined by Siegel in $[10 ; 11]$, the first two terms above are zero, since there are no automorphic forms associated to the factor of automorphy $\Delta^{-1}$; so that the cohomology sequence begins $0 \rightarrow H^{0}(\Gamma, \Xi) \rightarrow H^{1}(\Gamma, \mathscr{K}) \rightarrow \cdots$. Here $\mathscr{K}$ is actually a 5 -dimensional complex vector space, as is evident from (5.14); hence, in principle, the group $H^{1}(\Gamma, \mathscr{K})$ can be calculated purely algebraically, then giving a bound on the dimension of the space of automorphic forms $H^{0}(\Gamma, \Xi)$.

Next there is the operator $\mathscr{D}_{9}$, as in (5.3), for $f=4$; this furnishes a $(5$-homomorphism

$$
\mathscr{D}_{9}: \Theta_{14)} \rightarrow \Theta_{\Delta^{4}} \text {. }
$$

The kernel of $\mathscr{D}_{9}$ is again an invariant subspace under the action of $(5 ;$ this kernel $\mathscr{K}$, as is clear from (3.11), consists of those five-dimensional vector-valued functions $\left\{f_{v}(z)\right\}$ satisfying the partial differential equation

$$
\begin{aligned}
0= & \partial^{2} f_{0} / \partial z_{11}^{2}-2 \partial^{2} f_{1} / \partial z_{11} \partial z_{12}+2 \partial^{2} f_{2} / \partial z_{11} \partial z_{22}+\partial^{2} f_{2} / \partial z_{11}^{2} \\
& -2 \partial^{2} f_{3} / \partial z_{12} \partial z_{22}+\partial^{2} f_{4} / \partial z_{22}^{2} .
\end{aligned}
$$

The image of $\mathscr{D}_{9}$ is quite readily seen to be all of $\Theta_{\Delta^{4}}$. Thus in this case the Eichler cohomology sequence begins

$$
0 \rightarrow H^{0}(\Gamma, \mathscr{K}) \rightarrow H^{0}\left(\Gamma, \Theta_{\{4\}}\right) \rightarrow H^{0}\left(\Gamma, \Theta_{\Delta^{4}}\right) \rightarrow H^{1}(\Gamma, \mathscr{K}) \rightarrow \cdots,
$$

for any subgroup $\Gamma \subset \mathfrak{G}$. The space $\mathscr{K}$ is too complicated to permit any simple calculation of its cohomology groups. 
Finally there is the operator $\mathscr{D}_{7}$ as in (5.12), providing a $\Gamma$-homomorphism

$$
\mathscr{D}_{7}: \Theta_{\Delta^{1 / 2}} \rightarrow \Theta_{\Delta^{5 / 2}} \text {. }
$$

All of the functions involved here are the ordinary scalar-valued functions, and this case furnishes the closest parallel to the simpler general situation in the case of one complex variable. The mapping $\mathscr{D}_{7}$ is seen fairly clearly to be onto the space indicated in (5.17); and the kernel $\mathscr{K}$ consists of those scalar functions $f(z)$ which satisfy the partial differential equation

$$
4 \partial^{2} f / \partial z_{11} \partial z_{22}-\partial^{2} f / \partial z_{12}^{2}=0,
$$

as is evident from (3.9). It should be noted that the differential operator (5.18) is the wave equation; and that the boundary of the Siegel upper half-plane consists of characteristics of this equation; this is a situation which had arisen earlier as well, in [6]. In this case the associated Eichler cohomology sequence begins: $0 \rightarrow H^{0}(\Gamma, \mathscr{K}) \rightarrow H^{0}\left(\Gamma, \Theta_{\Delta^{1 / 2}}\right) \rightarrow H^{0}\left(\Gamma, \Theta_{\Delta^{5 / 2}}\right) \rightarrow H^{1}(\Gamma, \mathscr{K})$, for any discrete subgroup $\Gamma \subset \mathfrak{G}$. For the symplectic modular group, in particular, there are no automorphic forms associated to the factor of automorphy $\Delta^{1 / 2}$, so that the beginning of this cohomology sequence $0 \rightarrow H^{0}\left(\Gamma, \Theta_{\Delta^{5 / 2}}\right) \rightarrow H^{1}(\Gamma, \mathscr{K}) \rightarrow \cdots$ is quite parallel to the one-dimensional case.

\section{BIBLIOGRA PHY}

1. H. Boerner, Darstellungen von Gruppen, Springer, Berlin, 1955.

2. G. Bol, Invarianten linearer Differentialgleichungen, Abh. Math. Sem. Univ. Hamburg 16 (1949), 1-28.

3. E. Cartan, Sur la structure des groupes infinis de transformations, Ann. Sci. Ecole Norm Sup. 21 (1904), 153-206; ibid. 22 (1905), 218-308.

4. Séminaire H. Cartan, 1957/58, Fonctions automorphes. [Especially Lecture 4 (Cartan) and Lectures 5-10 (Godement)].

5. R. C. Gunning, Eichler cohomology groups and automorphic forms, Trans. Amer. Math. Soc. 100 (1961), 44-62.

6. - Generalized symplectic differential forms and differential operators, J. Math. Mech. 11 (1962), 703-724.

7. M. Koecher, Positivitätsbereiche im $R^{n}$, Amer. J. Math. 79 (1957), 575-596.

8. F. D. Murhaghan, The analysis of the direct product of irreducible representations of the symmetric groups, Amer. J. Math. 60 (1938), 44-65.

9. O. S. Rothaus, Domains of positivity, Abh. Math. Sem. Univ. Hamburg 24 (1960), 189-235.

10. C. L. Siegel, Einführung in die Theorie der Modulfunktionen n-ten Grades, Math. Ann. 116 (1939), 617-657.

11. - Symplectic geometry, Amer. J. Math. 65 (1943), 1-87.

12. H. Weyl, The classical groups, Princeton Univ. Press, Princeton, N. J., 1946.

Princeton UnIVERSTTY,

Princeton, New Jersey 